\title{
STOCHASTIC ORDERINGS INDUCED BY STAR-SHAPED FUNCTIONS
}

by

\author{
HENRY A. KRIEGER
}

Department of Mathematics

Harvey Mudd College

Claremont, CA 91711 USA

Abstract

(Received September 18, 1990)

The non-decreasing functions which are star-shaped and supported above at each point of a non-empty closed proper subset of the real line induce an ordering, on the class of distribution functions with finite first moments, that is strictly weaker than first degree stochastic dominance and strictly stronger than second degree stochastic dominance. Several characterizations of this ordering are devcloped, both joint distribution criteria and those involving only marginals. The latter are deduced from a decomposition theorem, which reduces the problem to consideration of certain functions which are star-shaped on the complement of an open interval.

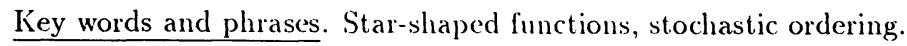
AMS 1980 Subject Classification. Primary 62E99, Secondary 60E05.

\section{Introduction}

In their study of selective risk aversion at a point $\mu \in \Re$, Landsberger and Meilijson [1] introduced the concept of a non-decreasing utility function which is star-shaped and supported above at this point $\mu$. More generally, they also considered non-decreasing utility functions which exhibit selective risk aversion at each point of an arbitrary non-empty closed proper subset $C$ of $\Re$, i.e., utility functions which are star-shaped and supported above at each point of this set $C$. Note that each such class of star-shaped function is contained in the class of non-decreasing functions, which induces the ordering known as first degree stochastic dominance on the collection of distribution functions (of probability measures on the Borel subsets of $\Re$ ). Moreover, each such class of star-shaped functions contains the class of nondecreasing concave functions, which induces the ordering known as second degree stochastic dominance on those distribution functions with finite first moments. Consequently, each of these classes of non-decreasing functions, star-shaped and supported above at each point of $C$, induces an ordering on the distribution functions with finite first moments that is strictly weaker than first degree stochastic dominance but strictly stronger than second degree stochastic dominance. 
In this paper we study these orderings, giving several necessary and sufficient conditions for two distribution functions to be so related to each other. We also consider additional hypotheses under which the necessary and sufficient condition can be simplified, simpler conditions which are sufficient but not necessary for the ordering to hold, special instances when the simpler sufficient conditions become necessary, and some examples to show that certain results cannot be improved.

\section{Some Special Classes of Star-shaped Functions and their Properties}

Let $U: \Re \rightarrow \Re$ be non-decreasing, star-shaped and supported above at a point $\mu \in \Re$. That is, $U(t)-U(\mu)=(t-u) S(t)$, where $S(t) \geq 0$ for all $t \in \Re, S(t)$ is non-increasing on $\Re$, but $(t-\mu) S(t)$ is non-decreasing on $\Re$. Let $\Pi$ be the collection of distribution functions, of probability measures on the Borel sets of $\Re$, with finite first moments.

A. Let $v=(\mu, \nu ; \xi ; \alpha, \beta, \gamma, \delta)$, where $\mu<\xi<\nu$ and $0<\alpha<\beta<\gamma<\delta$. Let $a$ and $b$ satisfy

$$
\begin{aligned}
& \delta(a-\mu)+\beta(\xi-a)=\gamma(\xi-\mu) \\
& \gamma(b-\xi)+\alpha(\nu-b)=\beta(\nu-\xi),
\end{aligned}
$$

so that $\mu<a<\xi<b<\nu$.

Define

$$
U_{v}(t)=\left\{\begin{array}{l}
\delta(t-\mu), t \leq a \\
\delta(a-\mu)+\beta(t-a), a \leq t \leq \xi \\
\delta(a-\mu)+\beta(\xi-a)+\gamma(t-\xi), \xi \leq t \leq b \\
\delta(a-\mu)+\beta(\xi-a)+\gamma(b-\xi)+\alpha(t-b), t \geq b .
\end{array}\right.
$$

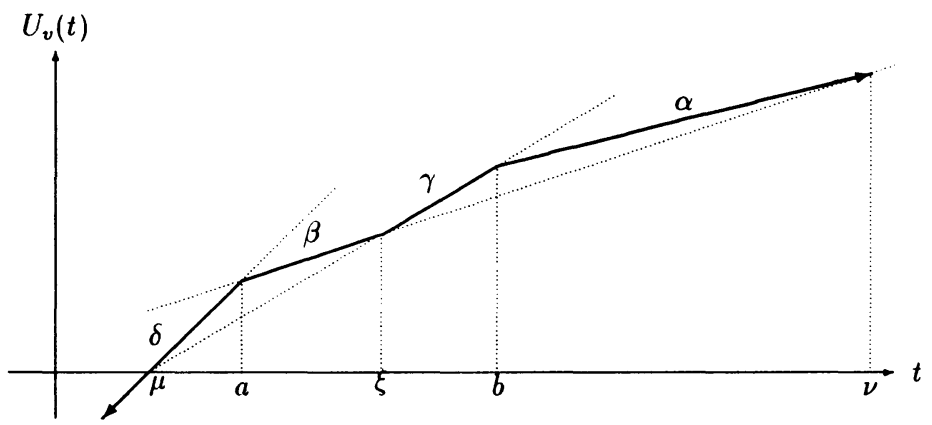

Properties of $U_{v}$ :

i) $U_{v}$ is strictly increasing of $\Re$, star-shaped and supported above at every point of $(-\infty, \mu] \cup[\nu, \infty)$ and at no point of $(\mu, \nu)$.

ii) $U_{v}$ is uniformly continuous on $\Re$, with $\left|U_{v}\left(t_{2}\right)-U_{v}\left(t_{1}\right)\right| \leq \delta\left|t_{2}-t_{2}\right|$, and $U_{v}(t) /(1+|t|)$ is bounded on $\Re$. 
Notes: if we relax the strict inequality $0<\alpha$ and allow $\alpha=0$, then $U_{v}$ is still non-decreasing on $\Re$ and retains the other properties. If we allow equality in any of the other strict inequalities $\alpha<\beta<\gamma<\delta$, then $U_{v}$ becomes concave on $\Re$.

Lemma 1. Let $\mu<x<\nu, Y \sim G \in \Pi$, and suppose that $E\left(U_{v}(Y)\right) \leq U_{v}(x)$ for all $v=(\mu, \nu ; \xi, \alpha, \beta, 1)$ where, $\xi, \alpha, \beta, \gamma$ are rational, $\mu<\xi<\nu$, and $0<\alpha<\beta<\gamma<1$. Then, in fact, $E\left(U_{v}(Y)\right) \leq U_{v}(x)$ for all $v=(\mu, \nu ; x ; \alpha, \beta, \gamma, \delta)$, where $\alpha, \beta, \gamma, \delta$ are real and $0 \leq \alpha \leq \beta \leq \gamma \leq \delta$, or equivalently.

$$
\gamma \int_{x}^{b}[1-G(t)] d t+\alpha \int_{b}^{\infty}[1-G(t)] d t \leq \delta \int_{-\infty}^{a} G(t) d t+\beta \int_{a}^{x} G(t) d t
$$

for all real $\alpha, \beta, \gamma, \delta$ with $0 \leq \alpha \leq \beta \leq \gamma \leq \delta$ and $a, b$ chosen to satisfy $\delta(a-\mu)+\beta(x-a)$ $=\gamma(x-\mu), \gamma(b-x)+\alpha(\nu-b)=\beta(\nu-x)$. Another one of the many equivalent forms is $(\gamma-\beta) \int_{x}^{\infty}[1-G(t)] d t \leq(\delta-\beta) \int_{-\infty}^{a} G(t) d t+(\gamma-\alpha) \int_{b}^{\infty}[1-G(t)] d t+\beta[x-E(Y)]$ for $0 \leq \alpha \leq \beta \leq \gamma \leq \delta$ with $(\delta-\beta)(a-\mu)=(\gamma-\beta)(x-\mu)$ and $(\gamma-\alpha)(b-x)=(\beta-\alpha)(\nu-x)$. Proof:

First note that for any $v=(\mu, \nu ; \xi, \alpha, \beta, \gamma, \delta)$, where $\xi \in(\mu, \nu)$ and $0 \leq \alpha \leq \beta \leq \gamma \leq \delta$, a direct calculation shows that

$$
E\left(U_{v}(Y)\right)-U_{v}(\xi)=\gamma \int_{\xi}^{b}[1-G(t)] d t+\alpha \int_{b}^{\infty}[1-G(t)] d t-\delta \int_{-\infty}^{a} G(t) d t-\beta \int_{a}^{\xi} G(t) d t
$$

where $a, b$ satisfy $\delta(a-\mu)+\beta(\xi-a)=\gamma(\xi-\mu), \gamma(b-\xi)+\alpha(\nu-b)=\beta(\nu-\xi)$. For emphasis, write $v(\xi)=(\mu, \nu ; \xi ; \alpha, \beta, \gamma, 1)$ and $v(x)=(\mu, \nu ; x ; \alpha, \beta, \gamma, 1)$ where $\xi, \alpha, \beta, \gamma$ are rational, $\xi \in(\mu, \nu)$ and $0<\alpha<\beta<\gamma<1$. Observe that for fixed $\alpha, \beta, \gamma, \delta$, with $0<\alpha<\beta<\gamma<\delta$, $a$ and $b$ are continuous functions of $\xi$. Moreover, $\left|U_{v(\xi)}(x)-U_{v(\xi)}(\xi)\right| \leq \delta|x-\xi| \rightarrow 0$ as $\xi \rightarrow x$. Consequently,

$$
\begin{aligned}
E\left(U_{v(x)}(Y)\right)-U_{v(x)}(x) & =\lim _{\xi \rightarrow x}\left[E\left(U_{v(\xi)}(Y)\right)-U_{v(\xi)}(\xi)\right] \\
& =\lim _{\xi \rightarrow x}\left[E\left(U_{v(\xi)}(Y)\right)-U_{v(\xi)}(x)\right] \\
& \leq 0,
\end{aligned}
$$

under our hypothesis. To conclude the proof, note that if $E\left(U_{v}(Y)\right) \leq U_{v}(x)$, then for any $\delta \geq 0$ the function $\delta U_{v}$ still satisfies this inequality. Finally, for fixed $x \in(\mu, \nu)$, the functions $a$ and $b$ defined by $\delta(a-\mu)+\beta(x-a)=\gamma(x-\mu), \gamma(b-x)+\alpha(\nu-b)=\beta(\nu-x)$ for $0<\alpha<\beta<\gamma<\delta$ remain well defined and continuous as long as either $0 \leq \alpha \leq \beta<\gamma \leq \delta$ or $0 \leq \alpha<\beta \leq \gamma<\delta$. The cases $\beta=\gamma=\delta$ and $\alpha=\beta=\gamma$ are independent of $a$ and $b$ respectively and can be verified directly. 
B. Consider the itcrated limit $\lim _{\beta|\cup| \cup} \lim _{\nu \mid \infty} U_{v}(t)$. Since $\nu \uparrow \infty$ implies $b \uparrow \infty$, we can denote this limit by $U_{v_{R}}(t)$, where $v_{R}=(\mu ; \xi ; \gamma, \delta)$ for $\mu<\xi$ and $0<\gamma<\delta$. Thus

$$
U_{v_{\mathcal{H}}}(t)=\left\{\begin{array}{l}
\delta(t-\mu), t \leq a \\
\delta(a-\mu), \quad a \leq t \leq \xi \\
\delta(a-\mu)+\gamma(t-\xi), t \geq \xi
\end{array}\right.
$$

where $a$ satisfies $\delta(a-\mu)=\gamma(\xi-\mu)$. In other words, $v_{R}=\left(\mu ; \xi ; \frac{(a-\mu)}{(\xi-\mu)} \delta, \delta\right)$ for $\mu<a<\xi$ and $\delta>0$.

Properties of $U_{v_{R}}$ :

i) $U_{v_{R}}$ is non-decreasing on $\Re$, star-shaped and supported above at each point of $(-\infty, \mu]$

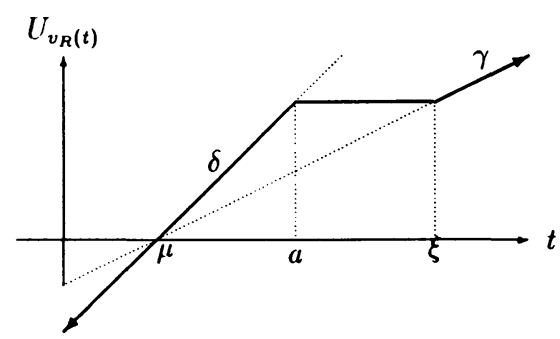

and at no point of $(\mu, \infty)$.

ii) $U_{v_{R}}$ is uniformly continuous on $\Re$, with $\left|U_{v_{R}}\left(t_{2}\right)-U_{v_{R}}\left(t_{1}\right)\right| \leq \delta\left|t_{2}-t_{1}\right|$, and $U_{v_{R}}(t) /(1+|t|)$ is bounded on $\Re$.

Note that if either of the strict inequalities $0<\gamma<\delta$ becomes an equality, then $U_{v_{R}}$ becomes concave on $\Re$.

Lemma 2. Let $\mu<x, Y \sim G \in \Pi$, and suppose that $E\left(U_{v_{R}}(Y)\right) \leq U_{v_{R}}(x)$ for all $v_{R}$ $=(\mu ; \xi ; \gamma, 1)$, where $\xi, \gamma$ are rational, $\mu<\xi$, and $0<\gamma<1$. Then, in fact, $E\left(U_{v_{R}}(Y)\right)$ $\leq U_{v_{R}}(x)$ for all $v_{R}=(\mu ; x ; \gamma, \delta)$, where $\gamma, \delta$ are real and $0 \leq \gamma \leq \delta$, or equivalently,

$$
\gamma \int_{x}^{\infty}[1-G(t)] d t \leq \delta \int_{-\infty}^{a} G(t) d t
$$

for all real $\gamma, \delta$ with $0 \leq \gamma \leq \delta$ and $a$ chosen to satisfy $\delta(a-\mu)=\gamma(x-\mu)$. Another equivalent form is $\frac{\int_{x}^{\infty}[1-G(t)] d t}{x-\mu} \leq \frac{\int_{-\infty}^{a} G(t) d t}{a-\mu}$ for $\mu<a \leq x$ or even $\mu<a<x$.

Proof: For any $v_{R}=(\mu ; \xi ; \gamma, \delta)$, where $\mu<\xi$ and $0 \leq \gamma \leq \delta$, either a direct calculation or two applications of the dominated convergence theorem to the iterated limit

$$
\lim _{\beta \backslash 0} \lim _{\nu \uparrow \infty}\left[E\left(U_{v}(Y)\right)-U_{v}(\xi)\right]
$$

shows that $E\left(U_{v_{R}}(Y)\right)-U_{v_{R}}(\xi)=\gamma \int_{\xi}^{\infty}[1-G(t)] d t-\delta \int_{-\infty}^{a} G(t) d t$, where $a$ satisfies $\delta(a-\mu)$ $=\gamma(\xi-\mu)$. The rest of the argument proceeds along the lines of the proof of Lemma 1.1 
C. Next, consider the iterated limit

$$
\lim _{\alpha \mid 0} \lim _{\mu \mid-\infty}\left[U_{v}(t)-U_{v}(\nu)\right]=\lim _{\alpha \mid 0} \lim _{\mu \mid-\infty}\left[U_{v}(t)-\gamma(\xi-\mu)-\beta(\nu-\xi)\right]
$$

Since $\mu \downarrow-\infty$ implies $a \downarrow-\infty$, we can denote this limit by $U_{v_{L}}(t)$, where $v_{L}=(\nu ; \xi ; \beta, \gamma)$ for $\xi<\nu$ and $0<\beta<\gamma$.

Hence

$$
U_{{ }^{\prime} L}(t)=\left\{\begin{array}{l}
\beta(t-\nu), t \leq \xi \\
\gamma(t-b), \xi \leq t \leq b \\
0, t \geq b
\end{array}\right.
$$

where $b$ satisfies $\gamma(b-\xi)=\beta(\nu-\xi)$. In other words, $v_{L}=\left(\nu ; \xi ; \beta, \frac{(\nu-\xi)}{(b-\xi)} \beta\right)$ for $\xi<b<\nu$ and $\beta>0$.

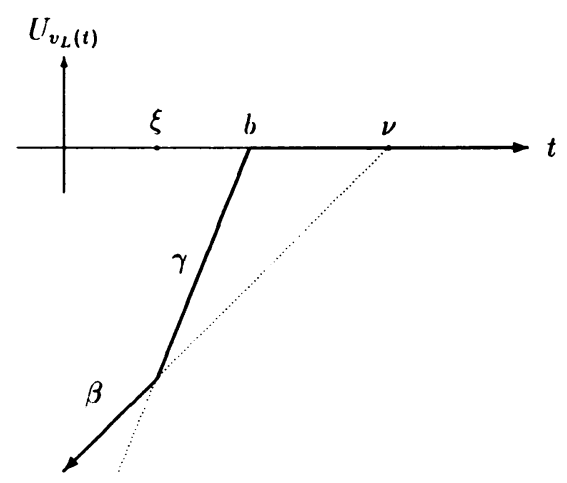

Properties of $U_{v_{L}}$ :

i) $U_{v_{L}}$ is non-decreasing on $\Re$, star-shaped and supported above at each point of $[\nu, \infty)$ and at no point of $(-\infty, \nu)$.

ii) $U_{v_{L}}$ is uniformly continuous on $\Re$, with $\left|U_{v_{L}}\left(t_{2}\right)-U_{v_{L}}\left(t_{1}\right)\right| \leq \gamma\left|t_{2}-t_{1}\right|$, and $U_{v_{L}}(t) /(1+|t|)$ is bounded on $\Re$.

Note that if either of the strict inequalities $0<\beta<\gamma$ is allowed to become an equality, then $U_{v_{L}}$ becomes concave on $\Re$.

Lemma 3. Let $x<\nu, Y \sim G \in \Pi$, and suppose that $E\left(U_{v_{L}}(Y)\right) \leq U_{v_{L}}(x)$ for all $v_{L}=(\nu ; \xi ; 1, \gamma)$, where $\xi, \gamma$ are rational, $\xi<\nu$, and $\gamma>1$. Then, in fact, $E\left(U_{v_{L}}(Y)\right) \leq U_{v_{L}}(x)$ for all $v_{L}=(\nu ; x ; \beta, \gamma)$, where $\beta, \gamma$ are real and $0 \leq \beta \leq \gamma$, or equivalently,

$$
\gamma \int_{x}^{b}[1-G(t)] d t \leq \beta \int_{-\infty}^{x} G(t) d t
$$

for all real $\beta$, $\gamma$ with $0 \leq \beta \leq \gamma$ and $b$ chosen to satisfy $\gamma(b-x)=\beta(\nu-x)$. An- 
other equivalent form is $\frac{\int_{x}^{b}[1-G(t)] d t}{b-x} \leq \frac{\int_{-x}^{x} G(t) d t}{\nu-x}$ for $x<b \leq \nu$ or even $x<b<\nu$. Proof: For any $v_{L}=(\nu ; \xi ; \beta, \gamma)$. where $\xi<\nu$ and $0 \leq \beta \leq \gamma$, either a direct calculation or two applications of the clominated convergence theorem to the iterated limit $\lim _{\alpha \backslash 0} \lim _{\mu \downarrow-\infty}\left\{E\left[U_{v}(Y)-U_{v}(\nu)\right]-\left[U_{v}(\xi)-I_{v}(\nu)\right]\right\}=\lim _{\alpha\rfloor 0} \lim _{\mu \backslash-\infty}\left[E\left(U_{v}\right)(Y)-\gamma(\xi-\mu)\right]$ shows that $E\left(U_{v_{L}}(Y)\right)-U_{v_{L}}(\xi)=\gamma \int_{\xi}^{b}[1-G(t)] d t-\beta \int_{-\infty}^{\xi} G(t) d t$, where $b$ satisfies $\gamma(b-\xi)=\beta(\nu-\xi)$.

The remainder of the argument again follow's the lines of the proof of Lemma 1.1

D. The final limiting form is $\lim _{\nu \mid \infty} U_{v_{L}}(t)=\lim _{\gamma \mid \infty} \lim _{\alpha \mid 0} \lim _{\mu \mid-\infty}\left[U_{v}(t)-\gamma(\xi-\mu)-\beta(\nu-\xi)\right]$. Since $\gamma \uparrow \infty$ implies $b \downarrow \xi$, we can denote the limit by $U_{v_{L L}}(t)$, where $v_{L L}=(\mu ; \xi ; \beta)$ for $\xi<\nu$ and $0<\beta$. Thus

$$
U_{v_{L L}}(t)=\left\{\begin{array}{l}
\beta(t-\nu), t \geq \xi \\
0, t>\xi
\end{array}\right.
$$

Properties of $U_{v_{L L}}$ :

i) $U_{v_{L L}}$ is non-decreasing on $\Re$, star-shaped and supported above at each point of $[\nu, \infty)$ and at no point of $(-\infty, \nu)$.

ii) $U_{v_{L L}}$ is discontinuous at $f$, although left continuous there, but continuous on the rest

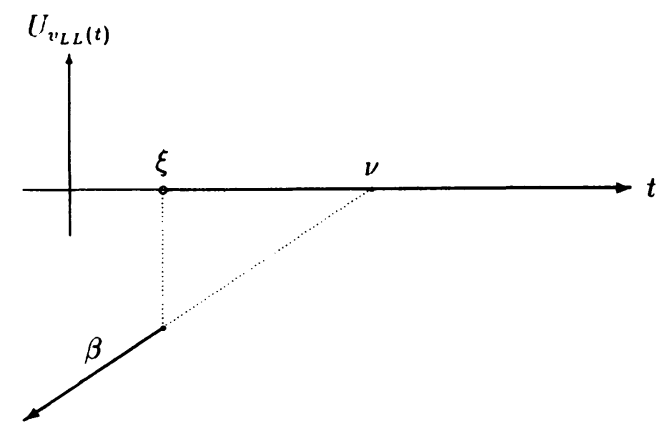

of $\Re$. It is still true that $U_{v_{L L}}(t) /(1+|t|)$ is bounded on $\Re$.

Note that if we allow $\beta=0$, then $U_{v_{L L}}$ becomes concave, in fact constant, on $\Re$.

Lemma 4. Let $x<\nu, Y \sim G \in \Pi$, and suppose that $E\left(U_{v_{L L}}(Y)\right) \leq U_{v_{L L}}(x)$ for all $v_{L L}=(\nu ; \xi ; 1)$, where $\xi$ is rational, $\xi<\nu$. Then, in fact, $E\left(U_{v_{L L}}(Y)\right) \leq U_{v_{L L}}(x)$ for all $v_{L L}=(\nu ; x ; \beta)$, where $\beta$ is real and $0 \leq \beta$, or equivalently, $\beta(\nu-x)[1-G(x)] \leq \beta \int_{-\infty}^{x} G(t) d t$ for all real $\beta \geq 0$. Other equivalent forms are $[1-G(x)] \leq \frac{\int_{-\infty}^{x} G(t) d t}{\nu-x}$ or $\int_{-\infty}^{x}(t-\nu) d G(t)$ $\leq x-\nu$.

Proof: For any $v_{L L}=(\nu ; \xi ; \beta)$, where $\xi<\nu$ and $0 \leq \beta$, either a direct calculation or an application of the dominated convergence theorem to $\lim _{\gamma \uparrow \infty}\left[E\left(U_{v_{L}}(Y)\right)-U_{v_{L}}(\xi)\right]$ shows that $E\left(U_{v_{L L}}(Y)\right)-U_{v_{L L}}(\xi)=\beta(\nu-\xi)[1-G(\xi)]-\beta \int_{-\infty}^{\xi} G(t) d t$. We can now proceed as in the proof of Lemma 1, except that we must approximate $x$ by rational $\xi>x$, since $G$ is only right continuous at $x$ and, for $v_{L L}=(\nu ; \xi ; 1), U_{v_{L L}}(\xi)-U_{v_{L L}}(x)=\xi-x$ for $\xi>x$. 


\section{Characterization of Distributions Which are No More Desirable than Certainty}

Let $C$ be a non-empty closed proper subset of $\Re$. Define $S_{C}$ to be the class of all nondecreasing functions on $\Re$ which are star-shaped and supported above at every point of $C$.

Proposition 1. Let $Y \sim G \in \Pi$ and $x \in \Re$. Then the following conditions are necessary and sufficient that $E\left(U\left(Y^{*}\right)\right) \leq U(x)$ for all $U \in S_{C}$.

i) If $x \in C: E(Y) \leq x$

ii) If $x \in(\mu, \nu)$ which is a bounded component of $C^{c}$ (the complement of $C$ ):

$$
\gamma \int_{x}^{b}[1-G(t)] d t+\alpha \int_{b}^{\infty}[1-G(t)] d t \leq \delta \int_{-\infty}^{a} G(t) d t+\beta \int_{a}^{x} G(t) d t
$$

for all real $a, \beta, \gamma, \delta$ with $0 \leq \alpha \leq \beta \leq \gamma \leq \delta$, where $a$ and $b$ satisfy

$$
\begin{aligned}
& \delta(a-\mu)+\beta(x-a)=\gamma(x-\mu), \\
& \gamma(b-x)+\alpha(\nu-b)=\beta(\nu-x) .
\end{aligned}
$$

iii) If $x \in(\mu, \infty)$ which is the component of $C^{c}$ unbounded above:

$$
\frac{\int_{x}^{\infty}[1-G(t)] d t}{x-\mu} \leq \frac{\int_{-\infty}^{a} G(t) d t}{a-\mu}
$$

for $\mu<a \leq x$.

iv) If $x \in(-\infty, \nu)$ which is the component of $C^{c}$ unbounded below:

$$
E(Y) \leq x \text { and }[1-G(x)] \leq \frac{\int_{-\infty}^{x} G(t) d t}{\nu-x}
$$

Proof: We begin with necessity. Since every non-decreasing concave function on $\Re$ belongs to $S_{C}, E(U(Y)) \leq U(x)$ for all $U \in S_{C}$ certainly implies $E(Y) \leq x$. Note that this condition is implicit in cases ii) and iii), taking $\alpha=\beta=\gamma=\delta>0$ and $a=x$ respectively. if $v=(\mu ; \nu ; x ; \alpha, \beta, \gamma, \delta)$, where $x \in(\mu, \nu)$ and $0 \leq \alpha \leq \beta \leq \gamma \leq \delta$, then the function $U_{v}$, being non-decreasing, star-shaped and supported above at every point of $(-\infty, \mu] \cup[\nu, \infty)$, certainly belongs to $S_{C}$. Since the condition of ii) is equivalent to $E\left(U_{v}(Y)\right) \leq U_{v}(x)$ for such a $v$, it must be necessary. Similarly, if $v_{R}=(\mu ; x ; \gamma, \delta)$, where $\mu<x$ and $0 \leq \gamma \leq \delta$, then $U_{v_{R}}$, being non-decreasing, star-shaped and supported above at each point of $(-\infty, \mu]$, belongs to $S_{C}$. But the condition of iii) is equivalent to $E\left(U_{v_{R}}(Y)\right) \leq U_{v_{R}}(x)$ for all such $v_{R}$, and hence must be necessary. Finally, let $v_{L L}=(\nu ; x ; \beta)$, where $x<\nu$ and $0 \leq \beta$. Then $U_{v_{L L}}$, being non-decreasing, star-shaped and supported above at all points of $[\nu, \infty)$, belongs 
to $S_{C}$. Since the condition of iv) is equiralent to $E(Y) \leq x$ and $E\left(U_{v_{L L}}(Y)\right) \leq U_{v_{L L}}(x)$, it also must be necessary:

For sufficiency in case i), we actually prove a slightly stronger result. Suppose $E(Y)$ $\leq \mu \leq x$ for some $\mu \in C$. Then, letting $\varepsilon=\mu-E(Y) \geq 0$, we have $E(U(Y)) \leq E(U(Y+\varepsilon))$ $\leq U(\mu) \leq U(x)$ for every $U \in S_{C}$, situce such a $U$ is non-decreasing on $\Re$ and supported above at $\mu=E\left(\nu^{\circ}+\varepsilon\right)$.

For case ii), suppose $x \in(\mu, \nu)$ and $U \in S_{C}$. Let $\alpha$ be the slope of a support line for $U$ at $(\nu, U(\nu)), \beta=\frac{L^{\prime}(\nu)-l^{\prime}(x)}{\nu-\alpha} \cdot \gamma=\frac{l^{\prime}(x)-U^{\prime}(\mu)}{x-\mu}$, and $\delta$ be the slope of a support line for $U$ at $(\mu, U(\mu))$. Then $0 \leq \alpha \leq \beta \leq \gamma \leq \delta$, since $U$ is non-decreasing on $\Re$ and star-shaped and supported above at both $\mu$ and $\nu$, and, hence, letting $v=(\mu, \nu ; x ; \alpha, \beta, \gamma, \delta)$, we have $U(t)-U(\mu) \leq U_{v}(t)$ for all $t \in \Re$ wilh equality for $\mu, x$, and $\nu$. But the condition of case ii) is equivalent to $E\left(l^{i}{ }_{v}(Y)\right) \leq l^{i}{ }^{\prime}(x)$ and, consequently, $\left.E(U(Y)) \leq U(\mu)+E\left(U_{v}\right)(Y)\right)$ $\leq U^{\prime}(\mu)+U_{v}(x)=U^{\prime}(x)$.

In case iii), suppose $x \in(\mu, \infty)$ and $U \in S_{C}$. Let $\gamma=\frac{U(x)-U(\mu)}{x-\mu}$ and $\delta$ be the slope of a support line for $U$ at $(\mu, U(\mu))$. Then $0 \leq \gamma \leq \delta$, since $U$ is non-decreasing on $\Re$ and starshaped and supported above at $\mu$, and if we let $v_{R}=(\mu ; x ; \gamma, \delta)$ we have $U(t)-U(\mu) \leq U_{v_{R}}(t)$ for all $t \in \Re$ with equality at $\mu$ and $x$. Since the condition of case iii) if equivalent to $E\left(U_{v_{R}}(Y)\right) \leq U_{v_{R}}(x)$, we have $E(U(Y)) \leq U(\mu)+E\left(U_{v_{R}}(Y)\right) \leq U(\mu)+U_{v_{R}}(x)=U(x)$.

Finally, for case iv), suppose $x \in(-\infty, \nu)$ and $U \in S_{C}$. Let $\alpha$ be the slope of a support line for $U$ at $(\nu, U(\nu))$ and $\beta=\frac{U(\nu)-U(x)}{\nu-x}$. Then $0 \leq \alpha \leq \beta$, since $U$ is non-decreasing on $\Re$ and star-shaped and supported above at $\nu$. Let $v_{L L}=(v ; x ; \beta-\alpha)$ and observe that $U(t)-U(v) \leq U_{v_{L L}}(t)+\alpha(t-v)$ for all $t \in \Re$ with equality for $x$ and $\nu$. But the condition of case iv) is equivalent to $E(Y) \leq x$ and $E\left(U_{v_{L L}}(x)\right) \leq U_{v_{L L}}(x)$. Therefore, we have $E(U(Y)) \leq U(\nu)+E\left(U_{v_{L L}}(Y)\right)+E(\alpha(Y-\nu)) \leq U(\nu)+U_{v_{L L}}(x)+\alpha(x-\nu)=U(x)$.

Corollary: Let $Y \sim G \in \Pi$ and $x=E(Y)$. Then the following conditions are necessary and sufficient that $E(U(Y)) \leq U(x)$ for all $U \in S_{C}$.

i) if $x \in C$ : the inequality holds for all such $Y$ and $U$.

ii) If $x \in(\mu, \nu)$, a bounded component of $C^{c}$ : any of the following holding for all $a, b$ such that $\mu<a \leq x \leq b<\nu$.

a) $\frac{(a-\mu)(\nu-b)}{(x-\mu)(\nu-x)} \int_{-\infty}^{x} G(t) d t \leq \frac{(\nu-b)}{(\nu-x)} \int_{-\infty}^{a} G(t) d t+\frac{(a-\mu)}{(x-\mu)} \int_{b}^{\infty}[1-G(t)] d t$

b) $\frac{(a-\mu)(\nu-b)}{(x-\mu)(\nu-x)} \int_{x}^{\infty}[1-G(t)] d t \leq \frac{(\nu-b)}{(\nu-x)} \int_{-\infty}^{a} G(t) d t+\frac{(a-\mu)}{(x-\mu)} \int_{b}^{\infty}[1-G(t)] d t$

c) $\frac{(a-\mu)(\nu-b)}{(\nu-x)} \int_{-\infty}^{x} G(t) d t+\frac{(a-\mu)(\nu-b)}{(x-\mu)} \int_{x}^{\infty}[1-G(t)] d t$ $\leq \frac{(\nu-b)(\nu-\mu)}{(\nu-x)} \int_{-\infty}^{a} G(t) d t+\frac{(a-\mu)(\nu-\mu)}{(x-\mu)} \int_{b}^{\infty}[1-G(t)] d t$

d) $\frac{(a-\mu)(\nu-b)}{(\nu-x)} \int_{a}^{x} G(t) d t+\frac{(a-\mu)(\nu-b)}{(x-\mu)} \int_{x}^{b}[1-G(t)] d t$ $\leq \frac{(\nu-b)(\nu-a)}{(\nu-x)} \int_{-\infty}^{a} G(t) d t+\frac{(a-\mu)(b-\mu)}{(x-\mu)} \int_{b}^{\infty}[1-G(t)] d t$. 
iii) If $x \in(\mu, \infty)$, the component of (" unbounded above: any of the following
a) $\frac{\int_{-\infty}^{u} C_{i}(t) d t}{u-\mu}$ non-increasing on $(\mu, r]$
b) $G^{\prime}\left(x^{-}\right) \leq \frac{\int_{-x}^{x} G_{i}(1) \cdot d !}{x-\mu}$
c) Either $P(Y=x)=1$ or $E^{\prime}(Y \mid Y<x) \leq \mu$.

iv) If $x \in(-\infty, \nu)$, the component of (" unbounded below: any of the following
a) $\frac{\int_{u}^{\infty}[1-G(t)] d t}{\nu-u}$ non-decreasing on $[x, \nu)$
b) $1-G(x) \leq \frac{\int_{1}^{\infty}[1-G(t)] d t}{\nu-x}$
c) Either $P\left(Y^{*}=x\right)=1$ or $E\left(Y^{\prime} \mid Y^{*}<x\right) \geq \nu$.

Proof: i) If $E(Y)=x \in C$, the necessary and sufficient condition of Proposition 1 is satisfied. ii) If $E(Y)=x \in(\mu, \nu)$, then

$$
\begin{aligned}
\int_{b}^{\infty}\left[1-G_{i}(t)\right] d t & =x-b+\int_{-\infty}^{b} G(t) d t \\
& =\int_{-\infty}^{a} C^{\prime}(t) d t+\int_{a}^{x} G(t) d t-\int_{x}^{b}[1-G(t)] d t
\end{aligned}
$$

so the necessary and sufficient condition of Proposition 1 becomes

$$
\begin{gathered}
(\gamma-\alpha) \int_{x}^{b}[1-G(t)] d t \leq(\delta-\alpha) \int_{-\infty}^{a} G(t) d t+(\beta-\alpha) \int_{a}^{x} G(t) d t \\
\text { or }(\gamma-\beta) \int_{-\infty}^{x} G(t) d t \leq(\delta-\beta) \int_{-\infty}^{a} G(t) d t+(\gamma-\alpha) \int_{b}^{\infty}[1-G(t)] d t
\end{gathered}
$$

for $0 \leq \alpha \leq \beta \leq \gamma \leq \delta$ where $a$ and $b$ satisfy $(\delta-\alpha)(a-\mu)+(\beta-\alpha)(x-a)=(\gamma-\alpha)(x-\mu)$ and $(\gamma-\alpha)(b-x)=(\beta-\alpha)(\nu-x)$. By continuity, we can assume $\beta<\gamma$ and in this case there is a one-one correspondence between $\frac{\beta-\alpha}{\delta-\alpha}$ and $\frac{\gamma-\alpha}{\delta-\alpha}$, on the one hand, and $a$ and $b$, where $\mu<a \leq x \leq b<\nu$, on the other hand. In fact, the solutions are

$$
\frac{\beta-\alpha}{\delta-\alpha}=\frac{(a-\mu)(b-x)}{(a-\mu)(\nu-x)+(x-a)(\nu-b)} \text { and } \frac{\gamma-\alpha}{\delta-\alpha}=\frac{(a-\mu)(\nu-x)}{(a-\mu)(\nu-x)+(x-a)(\nu-b)}
$$

Substituting these expressions into the last form of the inequality gives

$$
\left.(a-\mu)(\nu-b) \int_{-\infty}^{x} G(t) d t \leq(x-\mu)(\nu-b) \int_{-\infty}^{a} G(t) d t+(a-\mu)\right)(\nu-x) \int_{b}^{\infty}[1-G(t)] d t
$$

which is equivalent to a) above. The other forms follow from a) by simple manipulations and the relation $\int_{-\infty}^{x} G(t) d t=\int_{x}^{\infty}[1-G(t)] d t$.

iii) If $E(Y)=x \in(\mu, \infty)$, the condition of Proposition 1 becomes $\frac{\int_{-\infty}^{x} G(t) d t}{x-\mu} \leq \frac{\int_{-\infty}^{a} G(t) d t}{a-\mu}$ for $a \in(\mu, x]$. But the function $\frac{\int_{-\infty}^{u} G(t) d t}{u-\mu}$ is continuous on $(\mu, x]$ with a left-hand derivative there given by $\frac{(u-\mu) G\left(u^{-}\right)-\int_{-\infty}^{u} G(t) d t}{(u-\mu)^{2}}=\frac{\int_{-\infty}^{u-}(t-\mu) d G(t)}{(u-\mu)^{2}}$. Since $\int_{-\infty}^{u^{-}}(t-\mu) d G(t)$ is non-decreasing on $(\mu, x]$, we see that $\frac{\int_{-\infty}^{u} G(t) d t}{u-\mu}$ is non-increasing on $(\mu, x]$ (which implies the condition of 
Proposition 1) if and only if its left-liand derivative is non-positive at $x$ (which is implied by the condition of Proposition 1). That is, if and only if $(x-\mu) G\left(x^{-}\right) \leq \int_{-\infty}^{x} G(t) d t$ or, equivalently, $\int_{-\infty}^{x^{-}}(t-\mu) d G_{i}^{\prime}(t) \leq 0$, i.c. either $P(Y=x)=1$ or $E(Y \mid Y<x) \leq \mu$. Hence, conditions a), b) and c) are equivalent to each other and to the condition of Proposition 1. iv) If $E(Y)=x \in(-\infty, \nu)$, the condition of Proposition 1 becomes $1-G(x) \leq \frac{\int_{x}^{\infty}[1-G(t) d t}{\nu-x}$, which is our condition b) for this case. The function $\frac{\int_{u}^{\infty}[1-G(t)] d t}{\nu-u}$ is continuous on $[x, \nu)$ and has a right-hand derivative there giren by

$$
\frac{-(\nu-u)[1-G(u)]+\int_{u}^{\infty}[1-G(t)] d t}{(\nu-u)^{2}}=\frac{\int_{u}^{\infty}(t-\nu) d G(t)}{(\nu-u)^{2}}
$$

Since $\int_{u}^{\infty}(t-\nu) d G_{i}(t)$ is non-decreasing on $[x, \nu)$, we see that $\frac{\int_{u}^{\infty}[1-G(t)] d t}{\nu-u}$ is non-decreasing on $[x, \nu)$ if and only if its right-hand derivative is non-negative at $x$. That is, if and only if $\int_{x}^{\infty}[1-G(t)] d t \geq(\nu-x)\left[1-G^{\prime}(x)\right]$ or, equivalently, $\int_{x}^{\infty}(t-\nu) d G(t) \geq 0$, i.e. either $P(Y=x)=1$ or $E(Y \mid Y>x) \geq \nu$.

\section{Comments and Supplements:}

A. If $Y \sim G \in \Pi$ and $x \in I$, where $I$ is an open proper subinterval of $\Re$, then the proof of Proposition 1 shows that $E(U(Y)) \leq U(x)$ for all $U \in S_{\partial I}$ if and only if $E(\dot{U}(Y)) \leq U(x)$ for all $U \in S_{I^{c}}$. For if $C^{\prime}=\partial I$ then $I$ is one of the components of $C^{c}$ and the necessary and sufficient conditions of Proposition 1 are equivalent to $E(U(Y)) \leq U(x)$ for certain functions $U \in S_{I^{c}}$.

B. Conversely, the fact that $S_{C} \subset S_{\{\eta\}}$ for every $\eta \in C$ gives sufficient conditions on $Y \sim G \in \Pi$ and $x \in \Re$ to satisfy $E(U(Y)) \leq U(x)$ for all $U \in S_{C}$, namely the conditions necessary and sufficient for $S_{\{\eta\}}$. For example:

1. It is sufficient that for some $\mu \in C$, with $\mu<x$, condition iii) of Proposition 1 is satisfied; that is $\frac{\int_{x}^{\infty}[1-G(t)] d t}{x-\mu} \leq \frac{\int_{-\infty}^{a} G(t) d t}{a-\mu}$ for $\mu<a \leq x$.

2. Similarly, it is sufficient that for some $\nu \in C$, with $x<\nu$, condition iv) of Proposition 1 is satisfied; that is $E(Y) \leq x$ and $[1-G(x)] \leq \frac{\int_{-\infty}^{x} G(t) d t}{\nu-x}$.

3. In particular, if $x \in(\mu, \nu)$ which is a bounded component of $C^{c}$, then either of these two conditions is sufficient.

4. In case $E(Y)=x$, the corresponding versions of these sufficient conditions can be written as in Landsberger and Meilijson [1]: Let $J_{x}=\{x\}$ if $P(Y=x)=1$, otherwise let $J_{x}$ be the interval $[E(Y \mid Y<x) . E(Y \mid Y>x)]$. Then $E(U(Y)) \leq U(x)$ for all $U \in S_{C}$ provided that $J_{x} \cap C \neq \phi$.

C. However, if $x \in(\mu, \nu)$ which is a bounded component of $C^{c}$, there are situations in which these sufficient conditions are necessary as well. 
1. If $P\left(Y^{*} \leq \mu\right)=0$. condition iv) is necessary.

Proof: Starting with the necessary and sufficient condition ii) of Proposition 1 and assuming $G(t)=0$ for $t \leq \mu$, we $\operatorname{lot} \delta \uparrow \propto$ which implies $a \downarrow \mu$ and gives

$$
\gamma \int_{x}^{b}\left[1-G^{\prime}(t)\right] d t+\Omega \int_{b}^{\infty}[1-G(t)] d t \leq \beta \int_{-\infty}^{x} G(t) d t
$$

where $0 \leq \alpha \leq \beta \leq \gamma$ and $b$ satisfies $\gamma(b-x)+\alpha(\nu-b)=\beta(\nu-x)$. Next, let $\alpha \downarrow 0$ so that for $0 \leq \beta \leq \gamma$ with,$(b-x)=\beta(\nu-x)$

$$
\gamma \int_{x}^{b}\left[1-G^{\prime}(t)\right] d t \leq \beta \int_{-\infty}^{x} G(t) d t .
$$

Finally, if we let $\gamma \uparrow \infty$, which implies $b \downarrow x$, we get

$$
[1-G(x)] \leq \frac{\int_{-\infty}^{x} G(t) d t}{\nu-x}
$$

Since $E(Y) \leq x$ was already necessary from condition ii), we see that condition iv) is also necessary in this situation. Note also that if $E(Y)=x$ as well as $P(Y \leq \mu)=0$, the corresponding version of condition iv) follows directly from version b) of condition ii) in the corollary to Proposition 1 , after dividing by $a-\mu$ and letting $a \downarrow \mu$.

2. If $E(Y)=x$ and $P\left(Y^{\cdot} \geq \nu\right)=0$, condition iii) is necessary.

Proof: Again start with version b) of condition ii) in the corollary to Proposition 1, but this time divide by $\nu-b$ and then let $b \uparrow \nu$.

3. Combining the last two remarks, we see that if $E(Y)=x$ and $P(\mu<Y<\nu)=1$, then for $x \in(\mu, \nu)$ we have $E(U(Y)) \leq U(x)$ for all $U \in S_{C}$ if and only if $P(Y=x)=1$.

Proof: If $E(Y)=x \in(\mu, \nu)$ with both condition iii) and condition iv) holding, the only possibility is $P(Y=x)=1$.

D. To show we cannot go beyond the above situations with respect to necessity of conditions iii) and iv), we conclude with two examples.

1. There exists a $Y \sim G \in I I$ and an interval $(\mu, \nu)$ with $E(Y)=x \in(\mu, \nu)$ such that $G$ satisfies condition ii) but does not satisfy either condition iii) or condition iv).

Proof: For convenience, we take $-\mu=\nu>0=x$ and let $Y$ be uniformly distributed on $[-L, L]$, where $L \in(\nu, 2 \nu)$. For such an $L$, we see that on the one hand $P(Y \leq \mu)>0$ and $P(Y \geq \nu)>0$ while on the other hand $E(Y \mid Y<0)=-\frac{L}{2}>-\nu=\mu$ and $E(Y \mid Y>0)=\frac{L}{2}<\nu$, so that neither condition iii) nor condition iv) is satisfied. Because of all the symmetry in this example, version d) of condition ii) in the corollary to Proposition 
1 reduces to

$$
(a+\nu) \int_{a}^{u}\left(i(t) d t \leq(\nu-a) \int_{-\infty}^{a} G(t) d t\right.
$$

for $-\nu \leq a \leq 0$. Since $G$ is the uniform distribution on $[-L, L]$, we get

$$
h(a)=(a+\nu) \int_{a}^{0} C(t) d t-(\nu-a) \int_{-\infty}^{a} G(t) d t=\frac{L(a+\nu)}{4}-\frac{\nu(a+L)^{2}}{2 L} .
$$

The maximum value of this quadratic function of $a$ occurs at the point $a_{0}=\frac{-L(4 v-L)}{4 \nu}$. Since $L \in(\nu, 2 \nu)$ we sec that $a_{0} \in\left(-\nu,-\frac{3}{4} \nu\right) \subset(-\nu, 0)$. The maximum value $h\left(a_{0}\right)=\frac{L}{32 \nu}\left[(L-4 \nu)^{2}-8 \nu^{2}\right]$. Thus $h\left(a_{0}\right) \leq 0$ as long as $L \in(\nu, 2 \nu)$ is sufficiently close to $2 \nu$, namely $2 \nu-L \leq \frac{2}{1+\sqrt{2}} \nu$. Consequently, for any such value of $L, G$ satisfies condition ii).

2. There exists a $Y \sim G \in \Pi$ and an interval $(\mu, \nu)$ with $\mu<E(Y)<x<\nu$ such that $P(Y \geq \nu)=0$ and $(i$ satisfies condition ii) but does not satisfy condition iii) or iv).

Proof: Again we let $-\mu=\nu>0=x$, but this time let $Y^{\prime}$ be uniformly distributed on $[-L, \nu]$, where $L \in(\nu, 2 \nu)$. For such an $L$ we see that on the one hand, $P(Y \geq \nu)=0$ and $E(Y)=\frac{\nu-L}{2} \in\left(-\frac{\nu}{2}, 0\right) \subset(-\nu, 0)$. On the other hand, we see that for such a uniform distribution condition iv) is certainly not satisfied for $L \in(\nu, \sqrt{2} \nu)$ since

$$
[1-G(0)]-\frac{\int_{-\infty}^{0} G(t) d t}{\nu-0}=\frac{\nu}{\nu+L}-\frac{L^{2}}{2 \nu(\nu+L)}=\frac{2 \nu^{2}-L^{2}}{2 \nu(\nu+L)}>0
$$

Turning to condition iii) we require

$$
h(a)=(a+\nu) \int_{0}^{\infty}[1-G(t)] d t-\nu \int_{-\infty}^{a} G(t) d t=\frac{\nu}{2(\nu+L)}\left[\nu(a+\nu)-(a+L)^{2}\right] \leq 0
$$

for $-\nu \leq a \leq 0$. In this case we find that the maximum value of $\frac{h(a)}{a+\nu}$ for $-\nu<a<0$ occurs at $a_{0}=-(2 \nu-L) \in(-\nu, 0)$ for $L \in(\nu, 2 \nu)$ and this value is given by $\frac{h\left(a_{0}\right)}{a_{0}+\nu}=\frac{\nu}{2(\nu+L)}(5 \nu-4 L)$. Therefore the maximum is positive and hence condition iii) is not satisfied if $L \in\left(\nu, \frac{5}{4} \nu\right)$. Finally, writing condition ii) in the form $(\nu-b) h(a) \leq(a+\nu) k(b)$ for $-\nu \leq a \leq 0 \leq b \leq \nu$, where $k(b)=\nu \int_{b}^{\infty}[1-G(t)] d t+b[0-E(Y)]=\frac{\nu(\nu-b)^{2}}{2(\nu+L)}+\frac{b}{2}(L-\nu)$, we claim it will be satisfied for $L \in\left(\nu, \frac{5}{4} \nu\right)$ which are sufficiently close to $\frac{5}{4} \nu$. To see this, we find that the minimum value of $\frac{k(b)}{\nu-b}$ for $0<b<\nu$ occurs at $b_{0}=\nu-\sqrt{L^{2}-\nu^{2}} \in\left(0, \frac{1}{4} \nu\right) \subset(0, \nu)$ if $L \in\left(\nu, \frac{5}{4} \nu\right)$. This minimum is given by $\frac{k\left(b_{0}\right)}{\nu-b_{0}}=\frac{1}{2} \sqrt{\frac{L-\nu}{L+\nu}}\left[2 \nu-\sqrt{L^{2}-\nu^{2}}\right]$. But then $\frac{h\left(a_{0}\right)}{a_{0}+\nu} \leq \frac{k\left(b_{0}\right)}{\nu-b_{0}}$ means $\nu(5 \nu-4 L) \leq \sqrt{L^{2}-\nu^{2}}\left[2 \nu-\sqrt{L^{2}-\nu^{2}}\right]$, or equivalently $(2 \nu-L)^{2} \leq 2 \nu \sqrt{L^{2}-\nu^{2}}$ and such inequalities clearly hold for $L \in\left(\nu, \frac{5}{4} \nu\right)$ which are close enough to $\frac{5}{4} \nu$. 


\section{Orderings of Distributions with Respect to Star-shaped Functions}

Let $S_{C}$ denote the functions which are non-decreasing on $\Re$, star-shaped and supported above at each point of $\left(\therefore\right.$, a non-empty closed proper subset of $\Re$. Let $\hat{S}_{C} \subset S_{C}$ consist of the identity function $I(t)=t$ and the following additional functions. For any bounded component of $C^{c}$, say $(\mu, \nu)$, include the class of all $U_{v}$ for $v=(\mu, \nu ; \xi ; \alpha, \beta, \gamma, 1)$, where $\xi, \alpha, \beta, \gamma$ are rational, $\xi \in(\mu, \nu)$, and $0<\alpha<\beta<\gamma<1$. If $C^{c}$ has a component unbounded above, say $(\mu, \infty)$, include the class of all $U_{v_{\mu}}$ for $v_{R}=(\mu ; \xi ; \gamma, 1)$, where $\xi, \gamma$ are rational, $\mu<\xi$, and $0<\gamma<1$. If $C^{c}$ has a component unbounded below, say $(-\infty, \nu)$, include the class of all $U_{v_{L}}$ for $v_{L}=(\nu ; \xi ; 1, \gamma)$, where $\xi$, $\gamma$ are rational, $\xi<\nu$, and $1<\gamma$. Note that since $C^{c}$ has at most a count able number of components, $\hat{S}_{C}$ is countable. Furthermore, each $U \in \hat{S}_{C}$ is continuous and has the property that $U(t) /(1+|t|)$ is bounded on $\Re$.

If $Y \sim G$ and $A \sim F$, where $(i$ and $F$ belong to $\Pi$, we say that $G$ is not more desirable than $F$ with respect to $S_{C}$, written $G \leq_{C} F$. if and only if $E(U(Y)) \leq E(U(X))$ for all $U \in S_{C}$. If $x \in \Re$ and $\varepsilon_{x}$ is the probability distribution concentrated at $x$, let

$$
\Pi_{x}=\left\{G \in \Pi: G \leq_{C} \varepsilon_{x}\right\}
$$

Given a Markov kernel $T: \Re \times \mathcal{B} \rightarrow \Re$, where $\mathcal{B}$ is the Borel subsets of $\Re$, let $G_{x}(t)$ $=T(x,(-\infty, t])$ for $(x, t) \in \Re^{2}$ and for each $x \in \Re$ let $T_{x}$ be a random variable with distribution function $G_{x}$. Then $T$ ' will be called an $S_{C}$-dilation if and only if $G_{x} \in \Pi_{x}$ for all $x \in \Re$, i.e. $G_{x}$ has a finite first moment and $E\left(U\left(T_{x}\right)\right) \leq U(x)$ for every $x \in \Re$ and $U \in S_{C}$.

Theorem 1: For a Markov kernel $T$, with $C_{\alpha} \in$ II for all $x \in \Re$, the following are equivalent:

a) $T$ is an $S_{C}$-dilation

b) For each $x \in \Re, G_{x}$ satisfies the appropriate condition of Proposition 1.

c) $E\left(U\left(T_{x}\right)\right) \leq U(x)$ for every $x \in \Re$ and $U^{\prime} \in \hat{S}_{C}$.

Proof: Since $\hat{S}_{C} \subset S_{C}$, we immediately have a) $\Rightarrow$ c). If we assume c) then, since the identity function belongs to $\hat{S}_{C}, E\left(T_{x}\right) \leq x$ for all $x \in \Re$. Consequently, for each $x \in \Re G_{x}$ satisfies the appropriate condition of Proposition 1 because of Lemmas 1, 2, and 4, after observing that the hypotheses of Lemma 3 imply (by letting $\gamma \uparrow \infty$ ) those of Lemma 4 . Finally, if b) holds then the sufficiency half of Proposition 1 gives a).

In order to proceed, we need to make use of a version of a theorem from Strassen's [2] important 1965 paper, a fundamental work with many interesting applications. First the terminology: Let $\hat{v}(x)=1+|x|$ for $x \in \Re$ and $\hat{\omega}(x, t)=\hat{v}(x)+\hat{v}(t)$ for $(x, t) \in \Re^{2}$. Let $C_{\hat{v}}(\Re)$ be the set of all continuous functions v defined on $\Re$ such that $v / \hat{v}$ is bounded. Similarly, let $C_{\hat{\omega}}\left(\Re^{2}\right)$ be the set of all continuous lunctions $\omega$ defined on $\Re^{2}$ such that $\omega / \hat{\omega}$ is bounded. 
Then $C_{\hat{v}}(\Re)$ and $\left(\dot{w}\left(\Re^{2}\right)\right.$ are Banach spaces with norms $\|v\|_{1}=\sup \{|v(x)| / \hat{v}(x): x \in \Re\}$ and $\|\omega\|_{2}=\sup \left\{|\omega(x, t)| / \hat{\omega}(x, t):(x, t) \in \mathbb{R}^{2}\right\}$. Letting $\Pi_{i}, i=1,2$, be the natural projections from $\Re^{2}$ onto $\Re$, it turns out that $v \in C_{\dot{v}}(\Re)$ if and only if $\nu \circ \Pi_{i} \in C_{\hat{\dot{\omega}}}\left(\Re^{2}\right)$. To see this, note that if $v \in C_{i \dot{v}}(\Re)$, then $|v(x)| \leq\|v\|_{1} \hat{v}(x) \leq\|v\|_{1} \hat{\omega}(x, t)$ for all $(x, t) \in \Re^{2}$, so that $v \circ \Pi_{i} \in C_{\dot{\omega}}\left(\Re^{2}\right)$ and $\left\|v \circ \Pi_{\imath}\right\|_{2} \leq\|v\|_{1}$. Conversely, if $v \circ \Pi_{i} \in C_{\hat{\omega}}\left(\Re^{2}\right)$, then

$$
|v(x)| \leq\left\|v \circ \Pi_{i}\right\|_{2} \hat{\omega}(x, 0) \leq 2\left\|v \circ \Pi_{i}\right\|_{2} \hat{v}(x)
$$

for all $x \in \Re$, so that $v \in C_{\dot{v}}(\Re)$ and $\|v\|_{1} \leq 2\left\|v \circ \Pi_{i}\right\|_{2}$. Thus, in fact, the norms of $v$ in $C_{\hat{v}}(\Re)$ and $v \circ \Pi_{t}$ in $C_{\dot{\omega}}\left(\Re^{2}\right)$ are equivalent. Now let $\Pi^{2}$ be the set of all probability measures $P$ on the Borel scts of $\Re^{2}$ such that $\int \hat{\omega} d P<\infty$, i.e. $\int(|x|+|t|) P(d x d t)<\infty$, equipped with the topology $\mathcal{T}$ generated by the lunctionals $P \rightarrow \int \omega d P$ for $\omega \in C_{\hat{\omega}}\left(\Re^{2}\right)$ (in other words, the relativized weak $-\star$ topology when $\Pi^{2}$ is considered as a subset of the dual space of $C_{\hat{\omega}}\left(\Re^{2}\right)$ ). Note that if $P \in \Pi^{2}$ then the marginals $P_{\imath}=P \circ \Pi_{i}^{-1} \in \Pi$, the Borel probability distributions on $\Re$ with finite first moment.

Strassen's Theorem 7. Let $\Lambda$ be a non-empty, $\mathcal{T}$-closed, convex subset of $\Pi^{2}$ and let $F$ and $G$ belong to $\Pi$. Then a necessary and sufficient condition for the existence of a probability measure $P$ in $\Lambda$ with marginals $F$ and $G$, i.e. with $F=P_{1}=P \circ \Pi_{1}^{-1}$ and $G=P_{2}=P \circ \Pi_{2}^{-1}$, is that $\int v(x) F(d x)+\int u(t) G(d t) \leq \sup \left\{\int[v(x)+u(t)] Q(d x d t): Q \in \Lambda\right\}$ for all $v$ and $u$ in $C_{\hat{v}}(\Re)$.

We can now combine Strassen's Theorem with our Theorem 1 to yield the next result.

Theorem 2: For probability distributions $F$ and $G$ in $\Pi$, the following are equivalent:

a) $G \leq_{C} F$

b) There exists an $S_{C}$-dilation $T$ with $T F=G$ (i.e. $\int T(x, A) F(d x)=G(A)$ for all $A \in \mathcal{B})$

c) On some probability space there are random variables $X \sim F$ and $Y \sim G$ such that for every $U \in S_{C}, E(U(Y) \mid X) \leq U(X)$ holds almost surely.

Proof: Since b) $\Rightarrow$ c) and c) $\Rightarrow$ a) are clear, it suffices to show a) $\Rightarrow$ b). So assume $G \leq_{C} F$ and define $\Lambda=\left\{Q \in \Pi^{2}: \int[U(t)-U(x)] v(x) Q(d x d t) \leq 0\right.$ for all $U \in \hat{S}_{C}$ and all bounded, continuous, $v \geq 0$. We claim that $\Lambda$ is precisely the set of probability measures $Q$ for which there exits a regular condition distribution (Markov kernel) $T$, related to the marginals of $Q$

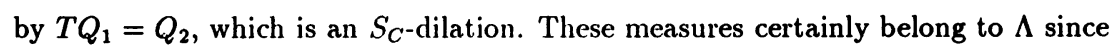

$$
\int[U(t)-U(x)] v(x) Q(d x d t)=\int\left\{\int[U(t)-U(x)] G_{x}(d t)\right\} v(x) Q_{1}(d x) \leq 0
$$


for all $U \in \hat{S}_{C}$ and bounded. continuous, non-negative $v$. Conversely, if $Q \in \Lambda$, with marginals $Q_{1}$ and $Q_{2}$, and $T^{\prime \prime}$ is any regular conditional distribution with $T^{\prime} Q_{1}=Q_{2}$, then $\int|t| G_{x}^{\prime}(d t)<\infty, Q_{1}$ almost surely, and for each $U \in \hat{S}_{C}$ we have $\int U(t) G_{x}^{\prime}(d t) \leq U(x), Q_{1}$ almost surely on the set where $G_{x}^{\prime \prime}$ has finite first moment. Since $\hat{S}_{C}$ is countable, there is a $Q_{1}$ null set $N$ such that for $x \notin N$ we have $\int|t| G_{x}^{\prime}(d t)<\infty$ and $\int U(t) G_{x}^{\prime}(d t) \leq U(x)$ for all $U \in \hat{S}_{C}$. Consequently, if we define $T(x, A)=T^{\prime}(x, A)$ for $x \notin N$ and $A \in \mathcal{B}$, with $T(x, A)=I_{A}(x)$ for $x \in N$ and $A \in \mathcal{B}$, we see that $T$ is an $S_{C}$-dilation (by Theorem 1) and $T Q_{1}=Q_{2}$.

Clearly, $\Lambda$ is non-empty and convex. Moreover, if $U \in \hat{S}_{C}$ then $U$ is continuous with $U(t) /(1+|t|)$ bounded on $\Re$. Thus if we let $\omega(x, t)=[U(t)-U(x)] v(x)$ for $U \in \hat{S}_{C}$ and $v$ bounded, continuous, and non-negative, then $\omega \in C_{\hat{\omega}}\left(\Re^{2}\right)$. In other words, $\Lambda$ is also a $\mathcal{T}$-closed subset of $\Pi^{2}$. By Strassen's Theorem, it remains to prove that for $v$ and $u$ in $C_{\hat{v}}(\Re)$ we have $\int v d F+\int u d G \leq \sup \left\{\int[v(x)+u(t)] Q(d x d t): Q \in \Lambda\right\}$.

Given $u \in C_{\hat{v}}(\Re)$, let $A_{u}=\left\{U \in S_{C}: U \geq u\right\}$. If $A_{u}=\emptyset$, let $u_{0}= \pm \infty$. Otherwise, let $u_{0}(t)=\inf \left\{U(t): U \in A_{u}\right\}$ for each $t \in \Re$. In any case, $u_{0} \geq u$. We claim that if $A_{u} \neq \emptyset$, then $u_{0} \in S_{C}$. To show $u_{0}$ is non-decreasing, let $t_{1}<t_{2}$. Then for every $U \in A_{u}$, $u_{0}\left(t_{1}\right) \leq U\left(t_{1}\right) \leq U\left(t_{2}\right)$, so that $u_{0}\left(t_{1}\right) \leq u_{0}\left(t_{2}\right)$. To show $u_{0}$ is supported above at any point $\mu \in C$, let $t_{1}<\mu<t_{2}$ with $\alpha \in(0,1)$ chosen so that $\alpha t_{1}+(1-\alpha) t_{2}=\mu$. Then for every $U \in A_{u}, \alpha u_{0}\left(t_{1}\right)+(1-\alpha) u_{0}\left(t_{2}\right) \leq \alpha U\left(t_{1}\right)+(1-\alpha) U\left(t_{2}\right) \leq U(\mu)$, so that $\alpha u_{0}\left(t_{1}\right)+(1-\alpha) u_{0}\left(t_{2}\right) \leq u_{0}(\mu)$. Finally, to show $u_{0}$ is star-shaped at any point $\mu \in C$, let $t \neq \mu$ and $\alpha \in(0,1)$. Then for every $U \in A_{u}, \alpha u_{0}(t)+(1-\alpha) u_{0}(\mu) \leq \alpha U(t)+(a-\alpha) U(\mu)$ $\leq U(\alpha t+(1-\alpha) \mu)$, and hence $\alpha u_{0}(t)+(1-\alpha) u_{0}(\mu) \leq u_{0}(\alpha t+(1-\alpha) \mu)$.

Therefore, if $v$ and $u$ belong to $C_{\hat{v}}(\Re)$, we have

$$
\int v d F+\int u d G \leq \int v d F+\int u_{0} d G \leq \int v d F+\int u_{0} d F
$$

since $G \leq_{C} F$ and either $u_{0} \in S_{C}$ or $u_{0}=+\infty$. Thus,

$$
\int v d F+\int u d G \leq \int\left[v+u_{0}\right] d F \leq \sup \left\{v(x)+u_{0}(x): x \in \Re\right\}
$$

So suppose we choose any $r<\sup \left\{v(x)+u_{0}(x): x \in \Re\right\}$. Strassen's condition will be satisfied if we can find a $Q \in \Lambda$ (depending upon $r$ ) such that $r<\int[v(x)+u(t)] Q(d x d t)$. To find such a $Q$ we need another function which dominates $u \in C_{\hat{v}}(\Re)$.

Recall that for $x \in \Re$, we defined $\Pi_{x}=\left\{H \in \Pi: H \leq_{C} \varepsilon_{x}\right\}$. Therefore, for $u \in C_{\hat{v}}(\Re)$ and $x \in \Re$, let $u_{1}(x)=\sup \left\{\int u d H: H \in \Pi_{x}\right\}$. Since $\varepsilon_{x} \in \Pi_{x}$, we have $u_{1}(x) \geq u(x)$ for all $x \in \Re$. Now suppose $x_{1}<x_{2}$. Since $U\left(x_{1}\right) \leq U\left(x_{2}\right)$ for every $U \in S_{C}$, we see that $\varepsilon_{x_{1}} \leq_{C} \varepsilon_{x_{1}}$. Since 
the relation $\leq_{C}$ is trausitive, we have $\Pi_{x_{1}} \subset \Pi_{x_{2}}$. But then for any $H \in \Pi_{x_{1}}, \int u d H \leq u_{1}\left(x_{2}\right)$ and hence $u_{1}\left(x_{1}\right) \leq u_{1}\left(x_{2}\right)$. Next, lat $\mu \in C^{\prime}$ and $x_{1}<\mu<x_{2}$ with $\alpha \in(0,1)$ chosen so that $\alpha x_{1}+(1-\alpha) \cdot x_{2}=\mu$. Since $\Omega^{I}\left(x_{1}\right)+(1-\alpha) U\left(x_{2}\right) \leq U(\mu)$ for all $U \in S_{C}$, we have $\alpha \varepsilon_{x_{1}}+(1-\alpha) \varepsilon_{x_{2}} \leq c \cdot \varepsilon_{\mu}$ and thus $a \Pi_{x_{1}}+(1-\alpha) \Pi_{x_{2}} \subset \Pi_{\mu}$. Therefore, if $H_{1} \in \Pi_{x_{1}}$ and $H_{2} \in \Pi_{x_{2}}$, we ser that $a / u d I_{1}+(1-a) \int u d I_{2} \leq u_{1}(\mu)$ and hence that $\alpha u_{1}\left(x_{1}\right)$ $+(1-\alpha) u_{1}\left(x_{2}\right) \leq u_{1}(\mu)$. At this point we can see that if $u_{1}(\mu)<\infty$ for any point $\mu \in C$, then $u_{1}(x)<\infty$ for all $x \in \Re, u_{1}$ is non-decreasing on $\Re$, and $u_{1}$ is supported above at all points $\mu \in C$. Finally, lot $\mu \in(;, x \neq \mu$. and $a \in(0,1)$. Since $\alpha U(x)+(1-\alpha) U(\mu)$ $\leq U(\alpha x+(1-a) \mu)$ for every $l^{i} \in S_{(}$. we get $a \varepsilon_{x}+(1-\alpha) \varepsilon_{\mu} \leq_{C} \varepsilon_{\alpha x+(1-\alpha) \mu}$. Thus $\alpha \Pi_{x}+(1-\alpha) \Pi_{\mu} \subset \Pi_{w^{2}+(1-\alpha) \mu}$. Therefore, if $H \in \Pi_{x}$ and $K \in \Pi_{\mu}$, we have $\alpha \int U d H$ $+(1-\alpha) \int u d K \leq u_{1}(\alpha x+(1-\alpha) \mu)$ and hence $\Omega u_{1}(x)+(1-\alpha) u_{1}(\mu) \leq u_{1}(\alpha x+(1-\alpha) \mu)$. We can now see that if $u_{1}(\mu)=+\infty$ for some $\mu \in C$, then $u_{1}(x)=+\infty$ for all $x \in \Re$. Thus we either have $u_{1}=+\infty$ or $u_{1} \in S_{C}$. But in the sccond case, this means $A_{u} \neq \emptyset$ and hence $u_{0} \in S_{C}$ with $u_{1} \geq u_{0}$. In any event, we certainly have $u_{1} \geq u_{0}$.

Now if $r<\sup \left\{v(x)+u_{0}(x): x \in \Re\right\}$, then for some $s \in \Re, r<v(s)+u_{0}(x) \leq v(s)+u_{1}(s)$. Since the inequality $r<v(s)+u_{1}(s)$ is equicalent to $r-v(s)<u_{1}(s)$, there exists an $H \in \Pi_{s}$ such that $r-v(s)<\int u d I I$ or, equivalently: $r<v(s)+\int u d H$. Now let $Q=\varepsilon_{s} \times H$. Then $Q \in \Lambda$ (taking $T(s, A)=H(A)$ and $T^{\prime}(x, A)=I_{A}(x)$ for $x \neq s, A \in \mathcal{B}$, for example) and $\int[v(x)+u(t)] Q(d x d t)=v(s)+\int u d H$.

\section{Further Conditions Necessary and/or Sufficient that $G \leq_{C} F$ :}

If $C$ is a non-empty closed proper subset of $\Re, Y \sim G$ and $X \sim F$, with $G$ and $F$ in $\Pi$, then we would like further characterizations of the relation $G \leq_{C} F$, i.e. $E(U(Y)) \leq E(U(X))$ for all $U \in S_{C}$. We begin with a decomposition theorem which reduces the problem to considering $U \in S_{I^{c}}$, where $I$ is a component of $C^{c}$. The basis for this theorem is the following lemma.

Lemma 5. Suppose $W \in S_{C}$ is linear on cach component of an open set (possibly empty) $J \subset \Re$. Let $I$ be a component of $C^{c}$ such that $I \cap J=\emptyset$. Then there exists functions $U$ and $V$ such that $U \in S_{l^{\circ}}$ is linear on each component of $\bar{I}^{c}, V \in S_{C}$ is linear on the components of $I \cup J$, and $W=U+V$. Moreover, if $I$ is bounded below with $\mu=$ glb $I$, then the slope of $U$ on $(-\infty, \mu)$ is $U^{\prime}\left(\mu^{+}\right)$.

Proof: If $I=(\mu, \infty)$ is unbounded above: let

$$
U^{\prime}(t)= \begin{cases}W^{\prime}(t), & t>\mu \\ W(\mu)+\delta(t-\mu), & t \leq \mu\end{cases}
$$


and

$$
r(t)= \begin{cases}0, & t>\mu \\ W(t)-W(\mu)-\delta(t-\mu), & t \leq \mu,\end{cases}
$$

where $\delta=W^{\prime}\left(\mu^{+}\right)$is the smallest support line slope for $W$ at $\mu$.

If $I=(-\infty, \nu)$ is unbounded below: let

$$
\zeta(t)= \begin{cases}0, & t \geq \nu \\ W^{\prime}(l)-W^{\prime}(\nu)-a(t-\nu), & t<\nu\end{cases}
$$

and

$$
V(t)= \begin{cases}\mathrm{W}^{\prime}(t), & t \geq \nu \\ \mathrm{W}^{\prime}(\nu)+\alpha(t-\nu), & t<\nu,\end{cases}
$$

where $\alpha=W^{\prime}\left(\nu^{+}\right)$is the smallest support line slope for $W$ at $\nu$.

If $I=(\mu, \nu)$ is bounded: let

$$
U(t)= \begin{cases}0, & t \geq \nu, \\ W(t)-W^{\prime}(\nu)-a(t-\nu), & \mu<t<\nu \\ W(\mu)-W(\nu)-a(t-\nu)+\delta(t-\mu), & t \leq \mu\end{cases}
$$

and

$$
V(t)= \begin{cases}W(t), & t \geq \nu \\ W(\nu)+a(t-\nu), & \mu<t<\nu \\ W(t)-W(\mu)+W(\nu)+\alpha(t-\nu)-\delta(t-\mu), & t \leq \mu\end{cases}
$$

where $\alpha=W^{\prime}\left(v^{+}\right)$and $\delta=W^{\prime}\left(\mu^{+}\right)$. Then it is casily checked that, in all cases, $W=U+V$ with $U$ and $V$ having the prescribed properties.

Theorem 3. Let $U \in S_{C}$ and let $\left\{I_{1}, I_{2}, \ldots\right\}$ be the components of $C^{\mathrm{c}}$. Then there exist functions $\left\{U_{0}, U_{1}, U_{2}, \ldots\right\}$ such that: $U_{0}$ is nou-decreasing, concave on $\Re$, and linear on the components of $C^{c}$; for each component $I_{m}$ of $C^{c}, U_{m} \in S_{I_{m}^{c}}$ and is linear on each component of $\bar{I}_{m}^{c}$; and $U=U_{0}+\sum_{m \geq 1} U_{m}$. Moreover, given $x \in \Re$, the functions $U_{m}$ for $m \geq 1$ can be chosen so that $U_{m}(x)=0$ and hence $U(x)=U_{0}(x)$.

Proof From Lemma 5, with $J=\emptyset$, we know that $U=U_{1}+V_{1}$, where $U_{1} \in S_{I_{1}^{c}}$ and is linear on each component of $\bar{I}_{1}^{c}, V_{1} \in S_{C}$ and is linear on $I_{1}$. So suppose that for some $n \geq 1$ we have $U=\sum_{m=1}^{n} L_{m}+V_{n}$, where $l_{m} \in S_{l_{m}^{k}}$ and is linear on each component of $\bar{I}_{m}^{c}$ for $m=1,2, \ldots, n, V_{n} \in S_{C}$ and is linear oll each component of $\cup_{m=1}^{n} I_{m}$. If $\cup_{m=1}^{n} I_{m}=C^{c}$, we stop. Otherwise, again by Lemma 5, this time with $J=\cup_{m=1}^{n} I_{m}$, we write $V_{n}=U_{n+1}+V_{n+1}$, where $U_{n+1} \in S_{I_{n+1}^{c}}$ and is linear on each component of $\bar{I}_{n+1}^{c}, V_{n+1} \in S_{C}$ and is linear on $\cup_{m=1}^{n+1} I_{m}$. Fixing $x \in \Re$, we see that the decomposition $U(t)=\sum_{m+1}^{n} U_{m}(t)+V_{n}(t)$ can be 
written $U(t)^{b}=\sum_{m=1}^{n}\left[U_{m}(t)-U_{m}(x)\right]+\left[V_{n}^{\prime}(t)-V_{n}^{\prime}(x)+U(x)\right]$, so we assume $U=\sum_{m=1}^{n} U_{m}+V_{n}$, where each $U_{m}(x)=0$ and $V_{n}^{\prime}(x)=I^{\prime}(x)$.

Now if $C^{c}$ has only a finite number of components we are finished, since in this case the last $V_{n}$, being linear on the componcuts of ('c and belonging to $S_{C}$, is concave on $\Re$ and hence we define $U_{0}$ to be this $V_{n}$. If $C^{\text {c }}$ lats an infinite number of components, we fix $x \in \Re$ and write $U=\sum_{m=1}^{n} U_{m}+V_{n}$, with all $U_{m}(x)=0$ and $V_{n}(x)=U(x)$, for every $n \geq 1$. But for each $t \geq x$, the secuence $\left(\sum_{m=1}^{n} U_{m}^{\prime}(t)\right)$ of partial sums of non-negative terms is bounded above by $U(t)-U^{\prime}(x)$ and hence the scquence $\left(V_{n}(t)\right)$ is non-increasing and bounded below by $U(x)$. Similarly, we see that for each $t<x$, the sequences $\left(\sum_{m=1}^{n} U_{m}(t)\right)$ and $\left(V_{n}(t)\right)$ are also convergent. Letting $U_{0}(t)=\lim _{n \rightarrow x} V_{n}(t)$, we sce that $U_{0}$ is linear on the components of $C^{c}$ and belongs to $S_{C^{\prime}}$, so that $U_{0}$ is concave on $\Re$. Thus $U=U_{0}+\sum_{m \geq 1} U_{m}$ gives the stated decomposition.

We now can see that the problem of chatacterizing $Y \sim G$ and $X \sim F$ with $G$ and $F$ in $\Pi$ for which $G \leq c \cdot F$. i.e. $\left.E\left(L^{\prime}()^{\circ}\right)\right) \leq E(U(X))$ for all $U \in S_{C}$, can be reduced to the corresponding problems for non-decreasing concave functions on $\Re$, where the result is well-known, and, for each componcut $I$ of $C^{c}$, the functions in $S_{I^{c}}$ which are linear on the components of $\bar{I}^{c}$. To complete the characterization in the case of bounded components and the case of a component unbounded above, we need to expand the special classes of star-shaped functions considered in section 2 .

A. Suppose $\mu<\nu, n \geq 2$, and $\delta_{1} \geq \delta_{2} \geq \cdots \geq \delta_{n} \geq 1 \geq \alpha_{1} \geq \alpha_{2} \geq \cdots \geq \alpha_{n} \geq 0$. Choose the points $\mu \leq a_{1} \leq x_{1} \leq a_{2} \leq \cdots \leq x_{n_{1}} \leq a_{n} \leq \nu$ so that

$$
\begin{array}{cr}
\delta_{\imath+1}\left(x_{\imath}-\mu\right)+\alpha_{1}\left(\nu-x_{\imath}\right)=\nu-\mu & \text { for } i=1,2, \cdots, n-1 \\
\text { and } \quad \delta_{i}\left(a_{i}-\mu\right)+\alpha_{\imath}\left(\nu-a_{\imath}\right)=\nu-\mu & \text { for } i=1,2, \cdots, n .
\end{array}
$$

Letting $x_{0}=-\infty$ and $x_{n}=+\infty$, we define $\dot{U}$ to be the piecewise-linear, continuous function with value 0 at $\mu$ and with slopes $\delta_{i}$ on $\left(x_{t-1}, a_{i}\right)$ for $i=1,2, \ldots, n$ and $\alpha_{i}$ on $\left(a_{i}, x_{i}\right)$ for $i=1,2, \ldots, n$. In other words

$$
\hat{U}(t)=\left\{\begin{array}{l}
\delta_{\imath}(t-\mu), t \in\left(x_{\imath-1}, a_{i}\right], i=1,2, \ldots, n \\
\alpha_{i}(t-\nu)+(\nu-\mu), t \in\left(a_{i}, x_{i}\right] i=1,2, \ldots, n .
\end{array}\right.
$$

Properties of $\tilde{U}$ :

i) $\tilde{U}$ is strictly increasing on $\Re$, star-shaped and supported above at every point of $(-\infty, \mu] \cup[\nu, \infty)$.

ii) $\tilde{U}$ is linear on $(-\infty, \mu)$ and on $(\nu, \infty)$.

iii) The case $n=2$ corresponds to the functions $U_{v}$ of section 2.A. 


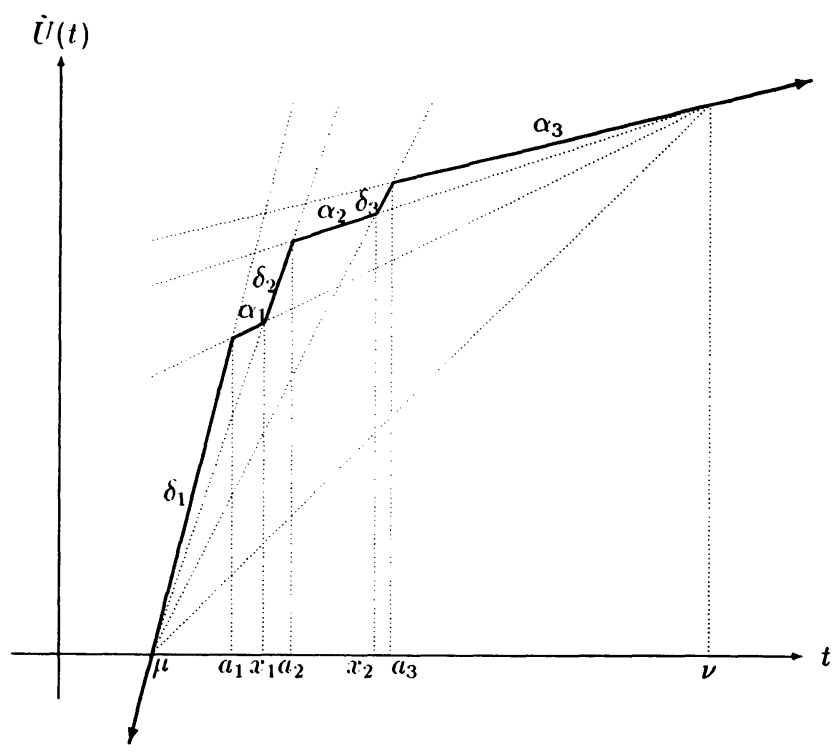

Lemma 6: If $Y \sim G, X \sim F$, with $G$ and $F$ in II, then

$$
E\left(\tilde{U}(Y)-E(\hat{U}(X))=-\sum_{i=1}^{n} \alpha_{i} \int_{a_{i}}^{x_{i}}[G(t)-F(t)] d t-\sum_{i=1}^{n} \delta_{i} \int_{x_{i-1}}^{a_{i}}[G(t)-F(t)] d t\right.
$$

Proof:

$$
\begin{gathered}
E(\tilde{U}(Y))-E\left(\tilde{U}\left(X^{\prime}\right)\right)=\sum_{i=1}^{n} \int_{a_{i}}^{x_{i}}\left[\alpha_{i}(t-\nu)+(\nu-\mu)\right][d G(t)-d F(t)] \\
+\sum_{i=1}^{n} \int_{x_{i-1}}^{a_{i}} \delta_{i}(t-\mu)[d G(t)-d F(t)]=\left.\sum_{i=1}^{n} \alpha_{i}(t-\nu)[G(t)-F(t)]\right|_{a_{i}} ^{x_{1}}-\sum_{i=1}^{n} \alpha_{i} \int_{a_{i}}^{x_{i}}[G(t)-F(t)] d t \\
\left.+(\nu-\mu) \sum_{i=1}^{n}[G(t)-F(t)]\right]_{a_{i}}^{x_{i}}+\left.\sum_{i=1}^{n} \delta_{i}(t-\mu)[G(t)-F(t)]\right|_{x_{i-1}} ^{a_{i}}-\sum_{i=1}^{n} \delta_{i} \int_{x_{i-1}}^{a_{i}}[G(t)-F(t)] d t \\
=-\sum_{i=1}^{n} \alpha_{i} \int_{a_{i}}^{x_{i}}[G(t)-F(t)] d t-\sum_{i=1}^{n} \delta_{i} \int_{x_{i-1}}^{a_{i}}[G(t)-F(t)] d t
\end{gathered}
$$

since

$$
\begin{gathered}
\left(x_{n}-\nu\right)\left[G\left(x_{n}\right)-F\left(x_{n}\right)\right]=\lim _{t \rightarrow \infty}(t-\nu)[G(t)-F(t)]=0, \\
\left(x_{0}-\mu\right)\left[G\left(x_{0}\right)-F\left(x_{0}\right)\right]=\lim _{t \rightarrow-\infty}(t-\mu)[G(t)-F(t)]=0, \\
\delta_{i+1}\left(x_{i}-\mu\right)+\alpha_{i}\left(\nu-x_{i}\right)=\nu-\mu
\end{gathered}
$$

for $i=1,2, \ldots, n-1$, and $\delta_{i}\left(a_{i}-\mu\right)+\alpha_{i}\left(\nu-a_{i}\right)=\nu-\mu$ for $i=1,2, \ldots, n$.

B. If we start with $\tilde{U}$ and successively let. $\nu \uparrow \infty$, which implies $a_{n} \uparrow \infty, \alpha_{1} \downarrow 0$, and then, for normalization, divide by $\delta_{1}$, we obtain a function $\tilde{\tilde{U}}$ which is determined for $n \geq 2$ by $1=\delta_{1} \geq \delta_{2} \geq \cdots \geq \delta_{n}>0$ and points $\mu \leq a_{1} \leq x_{1} \leq a_{2} \leq \cdots \leq a_{n-1} \leq x_{n-1}$ such that $\delta_{i}\left(a_{i}-\mu\right)=\delta_{i+1}\left(x_{i}-\mu\right)$ is non-decreasing for $i=1,2, \ldots, n-1$. Letting $x_{0}=-\infty$ and 
$a_{n}=+\infty$, we sec that $\dot{l}$ is the piecewise-lincar, continuous function with value 0 at $\mu$ and with slopes $\delta_{i}$ on $\left(x_{i-1}, a_{i}\right)$ for $i=1.2 \ldots \ldots$ and 0 on $\left(a_{i}, x_{i}\right)$ for $i=1,2, \ldots, n-1$. In other words,

$$
\dot{l}(t)=\left\{\begin{array}{l}
\delta_{i}(t-\mu), t \in\left(x_{i-1}, a_{i}\right], i=1,2, \ldots, n \\
\delta_{i}\left(a_{i}-\mu\right), t \in\left(a_{i}, x_{i}\right] i=1,2, \ldots, n-1 .
\end{array}\right.
$$

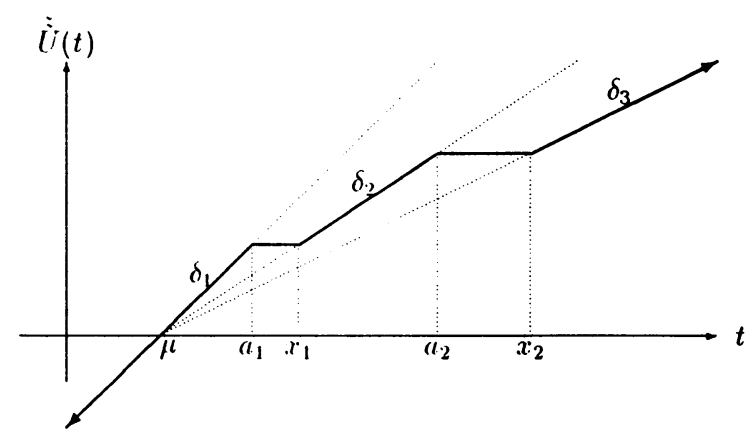

Properties of $\dot{\tilde{U}}$ :

i) $\tilde{\tilde{U}}$ is non-decreasing on $\Re$, star-shap)ed and supported above at every point of $(-\infty, \mu]$.

ii) $\tilde{\tilde{U}}$ is linear on $(-\infty, \mu)$.

iii) The case $n=2$ corresponds to the function $U_{v_{R}}$ of section 2.B.

Lemma 7: If $Y \sim G, X \sim F$, with $G$ and $F$ in II, then

$$
E(\dot{\dot{U}}(\dot{Y}))-E(\dot{\dot{U}}(\mathcal{X}))=-\sum_{i=1}^{n} \delta_{i} \int_{x_{i-1}}^{a_{i}}[G(t)-F(t)] d t .
$$

Proof: We can either use the result of Lemma 6, along with the dominated convergence theorem, or we can proceed directly. In the latter approach, we see that

$$
\begin{gathered}
E(\dot{\tilde{U}}(Y))-E(\dot{U}(X))=\sum_{i=1}^{n-1} \delta_{i}\left(a_{i}-\mu\right) \int_{a_{i}}^{x_{i}}[d G(t)-d F(t)] \\
+\sum_{i=1}^{n} \delta_{i} \int_{x_{i-1}}^{a_{i}}(t-\mu)[d G(t)-l F(t)]=\left.\sum_{i=1}^{n-1} \delta_{i}\left(a_{i}-\mu\right)[G(t)-F(t)]\right|_{a_{i}} ^{x_{i}} \\
\left.+\sum_{i=1}^{n} \delta_{i}(t-\mu)[G(t)-F(t)]\right]_{x_{i-1}}^{a_{i}}-\sum_{i=1}^{n} \delta_{i} \int_{x_{i-1}}^{a_{i}}[G(t)-F(t)] d t \\
=-\sum_{i=1}^{n} \delta_{i} \int_{x_{i-1}}^{a_{i}}[G(t)-F(t)] d t
\end{gathered}
$$

since $\left(a_{n}-\mu\right)\left[G\left(a_{n}\right)-F\left(a_{n}\right)\right]=\lim _{t \rightarrow \infty}(t-\mu)[G(t)-F(t)]=0,\left(x_{0}-\mu\right)\left[G\left(x_{0}\right)-F\left(x_{0}\right)\right]$ $=\lim _{t \rightarrow-\infty}(t-\mu)[G(t)-F(t)]=0$, and $\delta_{i+1}\left(x_{i}-\mu\right)=\delta_{i}\left(a_{i}-\mu\right)$ for $i=1,2, \ldots, n-1$.

Theorem 4. Let $Y \sim G, X \sim F$, where $G$ and $F$ belong to $\Pi$. Then $G \leq_{C} F$, i.e. 
$E(U(Y)) \leq E(U(X))$ for all $\| \in \in S_{c}$, if and only if all of the following conditions are satisfied:

i) $\int_{-\infty}^{x} G(t) d t \geq \int_{-\infty}^{x} F(t) d t$ for erery $x \in R$.

ii) For every bounded componcent of (", say" $(\mu, \nu)$, whenever

$$
\delta_{1} \geq \delta_{2} \geq \cdots \geq \delta_{n} \geq 1 \geq a_{1} \geq \alpha_{2} \geq \cdots \geq \alpha_{n} \geq 0
$$

$n \geq 2$, and $-\infty=x_{0}<\mu \leq a_{1} \leq x_{1} \leq a_{2} \leq \cdots \leq x_{n-1} \leq a_{n} \leq \nu<x_{n}=\infty$ satisfy $\delta_{i+1}\left(x_{i}-\mu\right)+a_{\imath}\left(\nu-x_{\imath}\right)=\nu-\mu$ for $i=1.2 \ldots, n-1, \delta_{\imath}\left(a_{\imath}-\mu\right)+\alpha_{i}\left(\nu-a_{\imath}\right)=\nu-\mu$ for $i=1,2, \ldots, n$, then $\sum_{i=1}^{n} \alpha_{i} \int_{a_{1}}^{x_{1}}[G(t)-F(t)] d t+\sum_{i=1}^{n} \delta_{i} \int_{x_{i-1}}^{a_{1}}[G(t)-F(t)] d t \geq 0$.

iii) For the componcnt of ("c unbounded above, say $(\mu, \infty)$, whenever

$$
1=\delta_{1} \geq \delta_{2} \geq \cdots \geq \delta_{n}>0
$$

$n \geq 2$, and $-\infty=x_{0}<\mu \leq a_{1} \leq x_{1} \leq a_{2} \leq \cdots \leq x_{n-1}<a_{n}=\infty$ satisfy $\delta_{\mathfrak{t}}\left(a_{\mathfrak{t}}-\mu\right)$ $=\delta_{t+1}\left(x_{i}-\mu\right)$ is non-decreasing for $i=1,2 \ldots, n-1$, then $\sum_{i=1}^{n} \delta_{i} \int_{x_{i-1}}^{a_{i}}[G(t)-F(t)] d t \geq 0$.

iv) For the component of $C^{c}$ unbounded below, say $(-\infty, \nu)$,

$$
\int_{-\infty}^{x}(t-\nu) d G(t) \leq \int_{-\infty}^{x}(t-\nu) d F(t) \text { for all } x<\nu .
$$

\section{Proof:}

If $U \in S_{c}$ and $H \in \Pi$, then $\int|U(t)| d H(t)<\infty$ if and only if $\int U(t) d H(t)>-\infty$. Furthermore, in the decomposition of $U$ given by Theorem 3 there is at most one term which might not have finite expectation with respect to $H$. If $C^{c}$ has a component unbounded below, then this term is the $U_{m}$ corresponding to that component. If there is no such component, then this term is $U_{0}$. In any case, even if $C^{c}$ has an infinite number of components, $-\infty \leq \int U d H=\int U_{v} d H+\sum_{m \geq 1} \int L_{m}^{\prime} d I I<\infty$. Consequently, $\int U d G \leq \int U d F$ for every $U \in S_{c}$ if and only if $\int I_{m} d G^{\prime} \leq \int l_{m} d F$ for all $l_{m}, m=0,1,2, \ldots$ of the type appearing in the decomposition. We claim that conditions i) through iv) collectively are both necessary and sufficient for all such inequalities to be satisficd.

If we define, for each $x \in \Re$, the non-decreasing concave function $U_{x}(t)=\left\{\begin{array}{l}t-x, t \leq x \\ 0, t>x,\end{array}\right.$ then condition i) is equivalent to $E\left(U_{x}\left(Y^{\prime}\right)\right) \leq E\left(U_{x}\left(X^{\prime}\right)\right)$ for every $x \in \Re$. But this is the wellknown necessary and sufficient condition that $E(U(Y)) \leq E(U(X))$ for all non-decreasing concave functions $U$ on $\Re$. 
Condition ii) is certainly necessary, since it is cquivalent, by Lemma 6 , to the statement that for every $\dot{U} \in S_{(\mu, \nu)^{*}} \subset S_{(\cdot,}$ w har $\left.l^{\prime}\left(\dot{l}()^{\circ}\right)\right) \leq E(\check{U}(X))$. We next show that this condition is sufficicut for $E^{\prime}\left(I^{\prime}\left(Y^{\prime}\right)\right) \leq E^{\prime}\left(l^{\prime}\left(X^{\prime}\right)\right)$ whenerer $U \in S_{(\mu, \nu)^{c}}, U$ is linear on $(-\infty, \mu)$ and $(\nu, \infty)$, and the slope of $l^{\prime}$ on $(-\infty . \mu)$ is $l^{\prime \prime}\left(\mu^{+}\right)$. We can assume that $\beta_{0}=\frac{U(\nu)-U(\mu)}{\nu-\mu}>0$, for otherwise $U(t)=I^{\prime}(\mu)$ for all $l \in R$. lior cacli $m \geq 1$, we define a $\tilde{U}_{m}$ as follows. Start with the points $x_{k, m}=\mu+(\nu-\mu) \frac{k}{2^{\prime \prime}}, k=1,2, \ldots, 2^{m}-1$. Next, let $\delta_{1, m}=\frac{U^{\prime}\left(\mu^{+}\right)}{\beta_{0}}$, $\delta_{k+1, m}=\frac{U\left(x_{k, m}\right)-l^{\prime}(\mu)}{\left(x_{k, m}-\mu\right) s_{0}^{\prime}}$ for $k=1,2 \ldots \ldots 2^{m}-1, a_{k, m}=\frac{U(\nu)-U\left(x_{k, m}\right)}{\left(\nu-x_{k, m}\right) \beta_{0}}$ for $k=1,2, \ldots, 2^{m}-1$, and $\alpha_{2^{m}, m}=\frac{u^{\prime \prime}\left(\nu^{+}\right)}{\beta_{0}}$. Observe that

$$
\delta_{1, m} \geq \delta_{2, m} \geq \ldots \geq \delta_{2^{m}, m} \geq 1 \geq \alpha_{1, m} \geq \alpha_{2, m} \geq \ldots \geq \alpha_{2^{m}, m} \geq 0
$$

and $\delta_{k+1, m}\left(x_{k, m}-\mu\right)+\alpha_{k, m_{l}}\left(\nu-x_{k, m}\right)=\frac{l(\nu)-U(\mu)}{\beta_{0}}=\nu-\mu$ for $k=1,2, \ldots, 2^{m}-1$. Therefore, these parameters define a function $\grave{U}_{m}$ which is readily seen to satisfy $\beta_{0} \tilde{U}_{m}(t)+U(\mu) \geq U(t)$ for every $t \in \Re$ with equality at the points $x_{k, m}, k=1,2, \ldots, 2^{m}-1$, and on $(-\infty, \mu] \cup[\nu, \infty)$. Moreover, since $U$ is continuous, we sec that as $m \uparrow \infty, \beta_{0} \tilde{U}_{m}(t)+U(\mu) \downarrow U(t)$ uniformly on $\Re$. Since we assume that $\int \dot{I}_{m} d C_{i} \leq \int \dot{I}_{m} d l^{\prime}$ for $m=1,2,3, \ldots$, we get $\int U d G \leq \int U d F$ as required.

Condition iii) is also necessary, since it is cquivalent, by Lemma 7, to the statement that for every $\tilde{\tilde{U}} \in S_{\left(\mu, x_{1}\right)} \subset S_{C}$, we have $\left.E\left(\dot{\tilde{U}}()^{\prime}\right)\right) \leq E\left(\tilde{\tilde{U}}\left(X^{\prime}\right)\right)$. We now show that the condition is sufficient for $E(U(Y)) \leq E(U(X))$ whenever $U \in S_{(\mu, \infty)^{c}}, U$ is linear on $(-\infty, \mu)$, and the slope $\delta_{0}$ of $U$ on $(-\infty, \mu)$ equals $U^{\prime}\left(\mu^{+}\right)$. We can assume that $\delta_{0}>0$, since otherwise $U(t)=U(\mu)$ for all $t \in \Re$. For each $m \geq 1$, we define a $\tilde{U}_{m}$ as follows. Let $x_{k, m}=\mu+\frac{k}{2^{m}}$ for $k=1,2, \ldots, m 2^{m}-1$. Then let $\delta_{1, m}=1$ and, for $k=1,2, \ldots, m 2^{m}-1$, let $\delta_{k+1, m}=\frac{U\left(x_{k, m}\right)-U(\mu)}{\left(x_{k, m}-\mu\right) \delta_{0}}$. Observe that $1=\delta_{1, m} \geq \delta_{2, m} \geq \ldots \geq \delta_{m 2^{m}, m}>0$ and $\delta_{k+1, m}\left(x_{k, m}-\mu\right)$ is non-decreasing as $k=1,2, \ldots, m 2^{m}-1$. Therefore, these parameters define a function $\tilde{\tilde{U}}$ which satisfies $\delta_{0} \tilde{U}(t)+U^{r}(\mu) \geq U^{\prime}(t)$ for every $t \in \Re$ with equality at the points $x_{k, m}$, $k=1,2, \ldots, m 2^{m}-1$, and on $(-\infty . \mu)$. Furthermore, since $U$ is continuous, we see that as $m \uparrow \infty, \delta_{0} \tilde{\tilde{U}}(t)+U(\mu) \downarrow U(t)$ pointwise on $\Re$ (actually uniformly on subsets of $\Re$ which are bounded above). Since we assume that $\int \hat{i}_{m} d G \leq \int \tilde{\tilde{U}}_{m} d F$ for $m=1,2,3, \ldots$, we see that $\int U d G \leq \int U d F$ as needed.

Finally, condition iv) is necessary, since it is equivalent, by an easy calculation, to $E\left(U_{v_{L L}}(Y)\right) \leq E\left(U_{v_{L L}}(X)\right)$ for all $v_{L L}=(\nu ; r ; 1)$, where $x<\nu$, and these functions belong to $S_{(-\infty, \nu)^{c}} \subset S_{c}$. We conclude by showing that this condition, along with condition i), is sufficient for $E(U(Y)) \leq E(U(X))$ whenever $U \in S_{(-\infty, u)^{c}}, U$ is linear on $(\nu, \infty)$, and $\int U d G>-\infty$. We write $U(t)-U(\nu)=S(t)(t-\nu)$, where $S$ is non-negative and non- 
increasing on $\Re$ with $S^{\prime}(l)=l^{\prime \prime}\left({ }^{+}\right)$for all $t \in(\nu, \infty)$. For $x \in \Re$, define $\hat{H}(x)$ $=\int_{-\infty}^{x}(t-\nu)\left[d G^{\prime}(t)-d F^{\prime}(t)\right]$. Thion condition i) tells us that $\hat{H}(\infty)=E(Y)-E(X) \leq 0$ and condition iv) is equivalent to $\hat{H}(x) \leq 0$ for $x<\nu$. Since $\hat{H}$ is continuous at $\nu$, we actually have $\hat{H}(\nu) \leq 0$ as woll. Now if $x \leq \nu$, thon $S^{\prime}(x) \int_{-\infty}^{x}(\nu-t) d G(t) \leq \int_{-\infty}^{x} S(t)(\nu-t) d G(t)$ $=\int_{-\infty}^{x}[U(\nu)-U(t)] d(i(t) \downarrow 0$ as $x \downarrow-\infty$. since $/ U d G>-\infty$. But then for $x \leq \nu$,

$$
0 \leq S(. x) \int_{-\infty}^{x}(\nu-l) d F(l) \leq S(x) \int_{-\infty}^{x}(\nu-t) d G(t)
$$

In particular, we ser that $\lim _{-\rightarrow \lambda} S(x) \hat{M}(x)=0$. ('onsequently,

$$
\begin{aligned}
\int U d C_{i}-\int l d F & =\int\left[l^{\prime}(l)-l(\nu)\right][d G(t)-d F(t)] \\
& =\int S(t)(t-\nu)[d G(t)-d F(t)] \\
& =\int S(t) d \hat{I I}(t) \\
& =S(+\infty) \hat{I}(+\infty)-\int_{-\infty}^{\infty} \hat{H}(t) d S(t) \\
& =l^{\prime \prime}\left(\nu^{+}\right)\left[U^{\prime}\left(Y^{+}\right)-E(X)\right]-\int_{-\infty}^{\nu^{+}} \hat{H}(t) d S(t) \\
& \leq 0 .
\end{aligned}
$$

since $U^{\prime}\left(\nu^{+}\right) \geq 0, E\left(Y^{\prime}\right) \leq E(X), \hat{H}(t) \leq$.0 for all $t \leq \nu$, and $S$ is non-increasing on $\Re$.

\section{Comments and Supplements:}

A. For $Y \sim G, X \sim F$, with $G$ and $F$ in II, let $G \leq_{1} F$ denote first order stochastic dominance, i.e. $C_{i}(x) \geq F(x)$ for all $x \in R$. and $G \leq_{2} F$ denote second order stochastic dominance, i.e. $\int_{-\infty}^{x}\left(i(l) d l \geq \int_{-\infty}^{x} f^{\prime}(l) d t\right.$ lor all $x \in \Re$. Then, because of characterizations of these orderings in terms of $E\left(U\left(I^{\circ}\right)\right) \leq E\left(I\left(X^{\prime}\right)\right)$ for all $U$ belonging to successively more restricted classes of functions on $\Re$, we sce that for any non-empty closed proper subset $C$ of $\Re, G \leq_{1} F \Rightarrow G \leq_{C} F \Rightarrow G \leq_{2} F$. Furthermore, neither of these implications is reversible, even if the distributions have equal means, as can be seen by Proposition 1 and its corollary for the simple case when $F=\varepsilon_{x}$.

B. For $Y \sim G, X \sim F$, with $G$ and $F$ in $I$, define as in Landsberger and Meilijson [3], for any $\mu \in \Re, G \leq_{\mu} F$ to mean $\int_{-\infty}^{x}(t-\mu) d G_{i}(t) \leq \int_{-\infty}^{x}(t-\mu) d F(t)$ for every $x \in \Re$. We claim that if $\mu \in C$ and $G \leq_{\mu} F$, then $G \leq_{C} F$.

Proof: As in the prool of Theorem 4 . we assume $U \in S_{C}$ with $\int U d G>-\infty$ and write $\int U d G-\int U d F=\int\left[U^{\prime}(t)-U(\mu)\right]\left[d C_{i}(t)-d F(t)\right]=\int S(t) d \hat{H}(t)$, where $S(t)$ is non-negative and non-increasing on $\Re, \hat{H}(x)=\int_{-\chi}^{r}(l-\mu)[d G(t)-d F(t)]$. Under the assumptions $\int U d G>-\infty$ and $\hat{H}(x) \leq 0$ for $x<\mu$, we showed that $\lim _{x \rightarrow-\infty} S(x) \hat{H}(x)=0$. Therefore, 
$\int U d G-\int U d F=S(+\infty) \hat{\Pi}(+\infty)-\int_{-\infty}^{\lambda} \hat{l}(I) d S(t) \leq 0$, since $\hat{H}(x) \leq 0$ for all $x \in \Re$ if $G \leq_{\mu} F$, so that $\hat{H}(+\infty)=\ell^{\prime}(Y)-\ell^{\prime}\left(X^{\prime}\right) \leq 0$ as well, and $0 \leq S(+\infty)<\infty$.

C. If $C=\{\mu\}$ and $E^{\prime}(Y)=E^{\prime}(\mathcal{X})$. lhen $\left(i \leq_{\mu} F^{\prime}\right.$ is also a necessary condition for $G \leq_{C} F$, see Landsberger and M/rilijson [:3].

Proof: Since we know that $\hat{\Pi}(x)>\int_{-\curlyvee}^{1}(l-\mu)[d G(t)-d F(t)] \leq 0$ for $x<\mu$, and thus for $x=\mu$ as well, is necessary, cren if $E^{\prime}\left(I^{\circ}\right)<l^{\prime}\left(X^{\circ}\right)$, we need only to show that this inequality must also hold for $x>\mu$ when $\left(i \leq_{c} F\right.$ and $E^{\prime}(Y)=E(X)$. So assume $\mu<x$ and consider

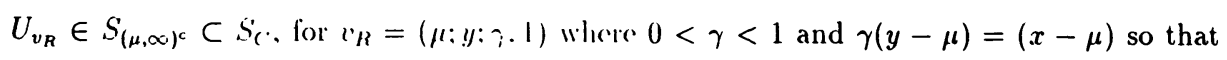

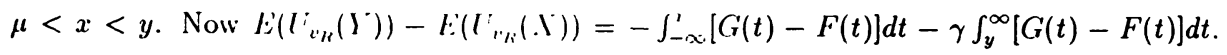
Hence if $\left.E\left(U_{v_{R}}()^{\circ}\right)\right) \leq E\left(L_{{ }^{*}{ }_{H}}\left(X^{*}\right)\right)$ and $\left.L^{\prime}()^{\circ}\right)=E\left(X^{*}\right)$, we get

$$
\begin{gathered}
-\gamma \int_{y}^{\infty}[G(t)-F(t)] d t=\gamma \int_{-\infty}^{y}\left[C^{\prime}(t)-F^{\prime}(t)\right] d t \leq \int_{\infty}^{x}[G(t)-F(t)] d t \text { or, equivalently, } \\
\gamma \int_{x}^{y}\left[C^{\prime}(t)-F^{\prime}(t)\right] d t \leq(1-\gamma) \int_{-\infty}^{x}[G(t)-F(t)] d t .
\end{gathered}
$$

Since $\gamma(y-\mu)=(x-\mu)$, we have $\frac{\int_{\perp}^{y}[F(t)-F(t)] d t}{y-J^{*}} \leq \frac{\int_{-\infty}^{x}[G(t)-F(t)] d t}{x-\mu}$. Letting $y \downarrow x$ gives $[G(x)-F(x)] \leq \frac{\int_{-\infty}^{x}[G(t)-F(t)] d t}{x-\mu}$ and this is c(quivalent to $\hat{H}(x) \leq 0$.

D. If $C=\{\mu\}$, but $E\left(Y^{\circ}\right)<E(X)$, then $C_{i} \leq_{\mu} F^{\prime}$ cannot be a necessary condition for $G \leq_{C} F$. Proof: Let $G=\varepsilon_{a}, F=\varepsilon_{b}$, where $\mu<a<b$. Then $G \leq_{1} F$, so that $G \leq_{C} F$, but for $a \leq x<b$ we have $\int_{-\infty}^{x}(t-\mu) d G(t)=a-\mu>0=\int_{-\infty}^{x}(t-\mu) d F(t)$.

E. If $E(Y)=E(X)$ but $C$ contains at least 2 points, say $\mu<\nu$, then neither $G \leq_{\mu} F$ nor $G \leq_{\nu} F$ can be a necessary condition for $G \leq_{C} F$.

Proof: First suppose that $(\mu, \nu)$ is a component of $C^{c}$. For convenience, we take $\mu=-\nu<0$ and, as in example 3.D.1, we let $G$ be the uniform distribution on $[-L, L]$ where $0<2 \nu-L \leq \frac{2}{1+\sqrt{2}} \nu$. If we let $F=\varepsilon_{0}$, then $G \leq_{C} F$. However,

$$
\begin{gathered}
\int_{-\infty}^{0^{-}}(t-\mu)[d G(t)-d F(t)]=(0+\nu)[C i(0)]-\int_{-\infty}^{0} G(t) d t=\frac{\nu}{2}-\frac{L}{4}>0 \text { and, similarly } \\
\int_{-\infty}^{0}(t-\nu)[d G(t)-d F(t)]=(0-\nu)[C(0)-1]-\int_{-\infty}^{0} G(t) d t=\frac{\nu}{2}-\frac{L}{4}>0 .
\end{gathered}
$$

If $(\mu, \nu)$ is not a component of $C^{c}$, then there must be a point $\eta \in(\mu, \nu)$ such that $\eta \in C$. Again, assuming for convenience that $\mu=-\nu<0$, the condition $\eta \in(\mu, \nu)$ is equivalent to $|\eta|<\nu$. Now let $G$ be the uniform distribution on $[\eta-L, \eta+L]$, where $\nu-|\eta|<L<2(\nu-|\eta|)$, and let $F=\varepsilon_{\eta}$. Since $\eta \in C$ and $G$ has mean $\eta$, the corollary to Proposition 1 shows that $G \leq_{C} F$. But $\int_{-\infty}^{\eta^{-}}(t-\mu)\left[d C^{\prime}(t)-d F^{\prime}(t)\right]=(\eta+\nu)\left[G^{\prime}(\eta)\right]-\int_{-\infty}^{\eta} G(t) d t=\frac{(\eta+\nu)}{2}-\frac{L}{4}>0$ and, similarly, $\int_{-\infty}^{\eta}(t-\nu)[d G(t)-d F(t)]=(\eta-\nu)[C(\eta)-1]-\int_{-\infty}^{\eta} G(t) d t=\frac{(\nu-\eta)}{2}-\frac{L}{4}>0$. 
F. Finally, suppose we have a function $l$ define on $\Re$ such that for every $Y \sim G \in \Pi$, $X \sim F \in \Pi$, with $\left(i \leq r \cdot l^{\prime}\left(l^{\prime}()^{\prime}\right)\right) \leq l^{\prime}\left(l^{\prime}\left(X^{\prime}\right)\right)$. Then the question arises: Does $U$ necessarily belong to $S_{4}$ ? The answer is ven.

Proof: Since $G \leq_{1} l \cdot$ sullices for $\left(i \leq_{1} \cdot F^{\prime}\right.$. wr take $\left(i=\varepsilon_{y}\right.$ and $F=\varepsilon_{x}$ when $y<x$ to see that $U(x) \leq U(y)$. i.e. $l i$ is non-decreasing on $R$. If $\mu \in C$, we let $x<\mu<y$ with $\alpha \in(0,1)$ chosen so that $\alpha \cdot x+(1-a) y=\mu .\left(i=a \varepsilon_{r}+(1-\alpha) \varepsilon_{y}\right.$, and $F=\varepsilon_{\mu}$. Then $G \leq_{C} F$ by Proposition 1 (or its corollary) since (i and $F$ both have expectation $\mu$. Therefore, $\alpha U(x)+(1-a) U(y) \leq I^{\prime}(\mu)$ and honc $/^{\prime}$ is supported above at $\mu$. Finally, suppose $\mu \in C$ $x_{0} \neq \mu$, and $\alpha \in(0,1)$. Let $G=\alpha \varepsilon_{\alpha_{0}}+(1-\alpha) \varepsilon_{\mu}$ and $F=\varepsilon_{\bar{x}}$, where $\bar{x}=\alpha x_{0}+(1-\alpha) \mu$. We thus have two cases, $x_{0}<\bar{x}<\mu$ and $\mu<\bar{x}<x_{0}$, and show in each case that $G \leq_{C} F$ because the sufficient (and necessary for $C^{\prime}=\{\mu\}$ since $G$ and $F$ have the same expectation) condition $G \leq_{\mu} F$ is satisficl. If $x_{0}<x<\mu$. Wr get

$$
\int_{-\infty}^{\lambda}(t-\mu)\left[d(i(t)-d l(t)]=\left\{\begin{array}{l}
0, \quad x<x_{0} \\
\vec{x}-\mu, \quad x_{0} \leq x<\bar{x} \\
0, \quad x \geq \bar{x}
\end{array}\right.\right.
$$

and if $\mu<\bar{x}<x_{0}$,

$$
\int_{-x}^{x}(t-\mu)\left[d C(i(t)-d F(t)]=\left\{\begin{array}{l}
0, \quad x<\bar{x} \\
\mu-\bar{x}, \quad \bar{x} \leq x<x_{0} \\
0, \quad x \geq x_{0}
\end{array}\right.\right.
$$

Thus, in either case, $\alpha U^{\prime}(x)+(1-\alpha) U^{\prime}(\mu) \leq U^{\prime}(\alpha x+(1-\alpha) \mu)$ which shows $U$ is star-shaped at $\mu$.ा 


\section{Acknowledgement:}

The author is very grateful to Prolessor lsalac Meilijson for his introduction to the subject of this paper and for many stimulating conversations in this regard. The research was conducted during a sabbatical leave spent with the Department of Statistics, School of Mathematical Sciences, Raymond and Bcrerly Sackler Faculty of Exact Sciences, Tel Aviv University, Israel.

\section{$\underline{\text { References }}$}

1. LANDSBERGER, M. and MEILI.JSON. I.: Lotteries, Insurance, and Star-shaped Utility Functions, Journal of Economic Throry, 52(19990), 65-82.

2. STRASSEN, V.: The Existence of Probability Measures with Given Marginals, Annals of Mathematical Statistics, 36(1965), 12:3-4:39).

3. LANDSBERGER, M. and MIEILIJSON. I.: A Tale of Two Tails: An Alternative Characterization of Comparative Risk, Joumul of Risk and Uncertainty, 3(1990), 65-82. 


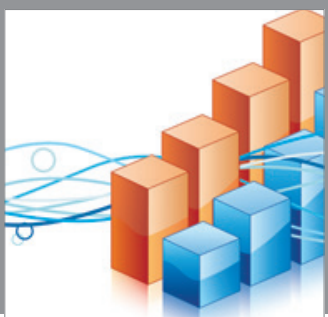

Advances in

Operations Research

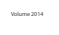

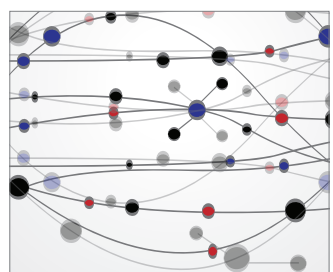

\section{The Scientific} World Journal
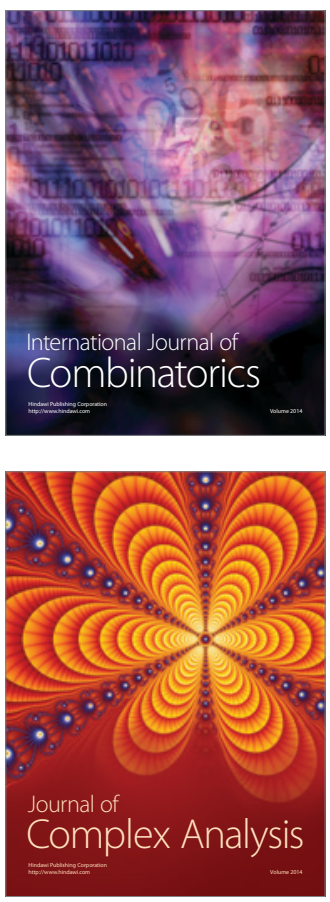

International Journal of

Mathematics and

Mathematical

Sciences
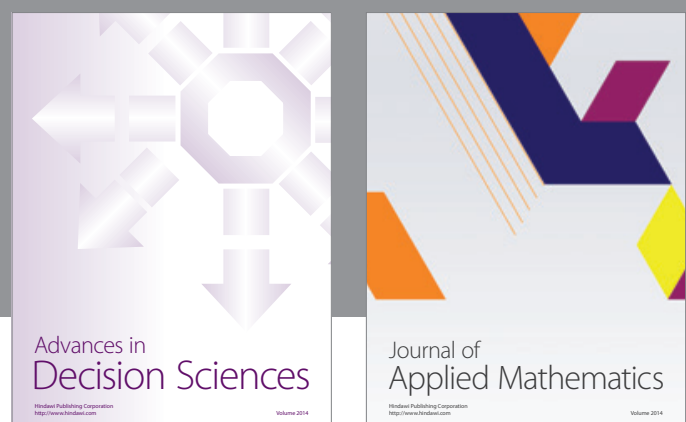

Journal of

Applied Mathematics
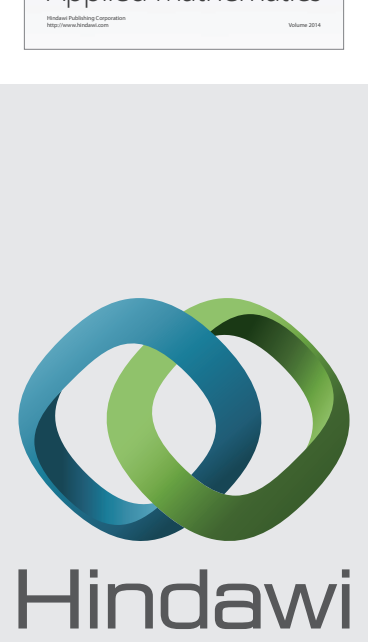

Submit your manuscripts at http://www.hindawi.com
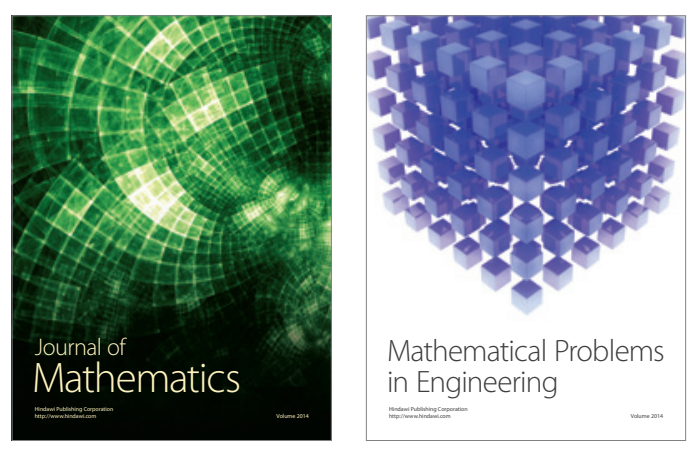

Mathematical Problems in Engineering
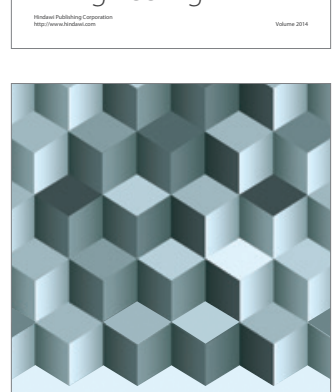

Journal of

Function Spaces
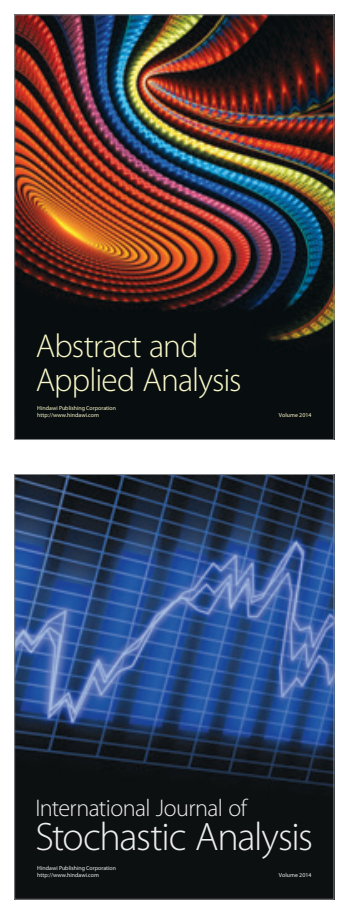

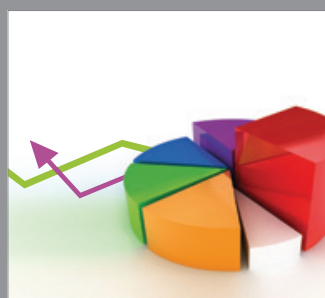

ournal of

Probability and Statistics

Promensencen
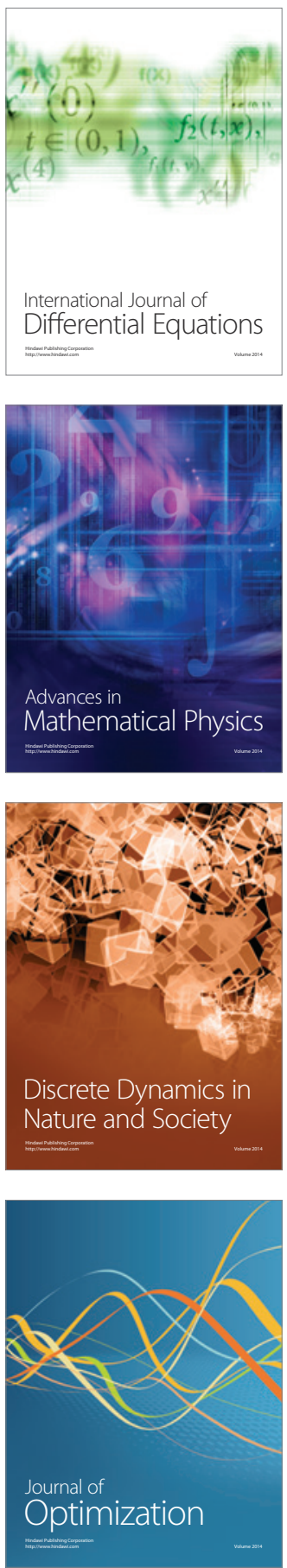